\section{SirT7 auto-ADP-ribosylation regulates glucose starvation response through $\mathrm{mH} 2 \mathrm{~A} 1$}

\author{
Nicolás G. Simonet ${ }^{1,2}$, Joshua K. Thackray ${ }^{3}$, Berta N. Vazquez ${ }^{1,2,3}$, Alessandro lanni ${ }^{4}$, \\ Maria Espinosa-Alcantud ${ }^{1,2}$, Julia Morales-Sanfrutos ${ }^{5,6}$, Sarah Hurtado-Bagès' ${ }^{7}$, Eduard Sabidó ${ }^{5,6}$, \\ Marcus Buschbeck ${ }^{7,8}$, Jay Tischfield ${ }^{3}$, Carolina De La Torre ${ }^{9,10}$, Manel Esteller ${ }^{11,12,13,14}$, \\ Thomas Braun $^{4}$, Mireia Olivella ${ }^{15,16}$, Lourdes Serrano ${ }^{3,17}$, Alejandro Vaquero ${ }^{1,2 *}$
}

\begin{abstract}
Sirtuins are key players of metabolic stress response. Originally described as deacetylases, some sirtuins also exhibit poorly understood mono-adenosine 5'-diphosphate (ADP)-ribosyltransferase (mADPRT) activity. We report that the deacetylase SirT7 is a dual sirtuin, as it also features auto-mADPRT activity. SirT7 mADPRT occurs at a previously undefined active site, and its abrogation alters SirT7 chromatin distribution. We identify an epigenetic pathway by which ADP-ribosyl-SirT7 is recognized by the ADP-ribose reader $\mathrm{mH} 2 \mathrm{~A} 1.1$ under glucose starvation, inducing SirT7 relocalization to intergenic regions. SirT7 promotes $\mathrm{mH} 2 \mathrm{~A} 1$ enrichment in a subset of nearby genes, many of them involved in second messenger signaling, resulting in their specific up- or down-regulation. The expression profile of these genes under calorie restriction is consistently abrogated in SirT7-deficient mice, resulting in impaired activation of autophagy. Our work provides a novel perspective on sirtuin duality and suggests a role for SirT7/mH2A1.1 axis in glucose homeostasis and aging.
\end{abstract}

\section{INTRODUCTION}

The members of the Sir2 family, also known as sirtuins, play a key role in the response to different types of stress, including metabolic, oxidative, and genotoxic stress. Thus, sirtuins are at the crossroads of many pathways that control genome stability, metabolic homeostasis, cell cycle control, and apoptosis (1). The family members originated in the prokaryotes, where they participated in the metabolism of nicotinamide adenine dinucleotide $\left(\mathrm{NAD}^{+}\right)$. During evolution, sirtuins have expanded and diversified, acquiring a key role in the cross-talk between chromatin and environment. Reflecting this, four of the seven mammalian sirtuins (SirT1, SirT2, SirT6, and SirT7) have been linked to the control of genome stability at different

\footnotetext{
${ }^{1}$ Chromatin Biology Laboratory, Josep Carreras Leukaemia Research Institute (IJC), Ctra de Can Ruti, Camí de les Escoles s/n, 08916 Badalona, Barcelona, Catalonia, Spain. ${ }^{2}$ Chromatin Biology Laboratory, Cancer Epigenetics and Biology Program (PEBC), Bellvitge Biomedical Research Institute (IDIBELL), Av. Gran Via de l'Hospitalet, 199-203, 08908 L'Hospitalet de Llobregat, Barcelona, Catalonia, Spain. ${ }^{3}$ Department of Genetics and the Human Genetics Institute of New Jersey, Rutgers University, 145 Bevier Road, Piscataway, NJ 08854, USA. ${ }^{4}$ Max Planck Institute for Heart and Lung Research, Department of Cardiac Development and Remodelling, Bad Nauheim, Germany. ${ }^{5}$ Proteomics Unit, Centre de Regulació Genòmica (CRG), Barcelona Institute of Science and Technology (BIST), Barcelona, Catalonia, Spain. ${ }^{6}$ Universitat Pompeu Fabra, Barcelona, Spain. 'Cancer and Leukemia Epigenetics and Biology Program, Josep Carreras Leukaemia Research Institute (IJC), Campus ICO-GTP-UAB, 08916 Badalona, Barcelona, Catalonia, Spain. ${ }^{8}$ Program for Predictive and Personalized Medicine of Cancer, Germans Trias i Pujol Research Institute (PMPPC-IGTP), 08916 Badalona, Barcelona, Catalonia, Spain. ${ }^{9}$ Proteomics Unit, Josep Carreras Leukaemia Research Institute (IJC), Ctra de Can Ruti, Camí de les Escoles s/n, 08916 Badalona, Barcelona, Catalonia, Spain. ${ }^{10}$ Proteomics Unit, Bellvitge Biomedical Research Institute (IDIBELL), Av. Gran Via de l'Hospitalet, 199-203, 08908 L'Hospitalet de Llobregat, Barcelona, Catalonia, Spain. ${ }^{11}$ Cancer Epigenetics Group, Josep Carreras Leukaemia Research Institute (IJC), Badalona, Barcelona, Catalonia, Spain. ${ }^{12}$ Centro de Investigación Biomédica en Red Cáncer (CIBERONC), 28029 Madrid, Spain. ${ }^{13}$ Institucio Catalana de Recerca i Estudis Avançats (ICREA), Barcelona, Catalonia, Spain. ${ }^{14}$ Physiological Sciences Department, School of Medicine and Health Sciences, University of Barcelona (UB), Barcelona, Catalonia, Spain. ${ }^{15}$ Bioinformatics Area, School of International Studies, ESCI-UPF, Barcelona, Catalonia, Spain. ${ }^{16}$ Bioinformatics and Medical Statistics Group, UST, Universitat de Vic-Universitat Central de Catalunya (UVIC-UCC), Barcelona, Catalonia, Spain. ${ }^{17}$ Department of Science, BMCC, The City University of New York (CUNY), 199 Chambers Street N699P, New York, NY 10007, USA.

*Corresponding author. Email: avaquero@carrerasresearch.org
}

levels (2). One of the most intriguing features of sirtuin biology is that they catalyze two $\mathrm{NAD}^{+}$-dependent enzymatic activities: $\mathrm{NAD}^{+}$dependent deacetylation and mono-adenosine $5^{\prime}$-diphosphate (ADP)ribosylation (mADPR), which involves the reversible transfer of one ADP-ribose moiety from the coenzyme $\mathrm{NAD}^{+}$to specific protein residues. The former seems to be more prominent among mammalian sirtuins since almost all of them are $\mathrm{NAD}^{+}$-dependent protein deacetylases, whose activity can, in some cases, target other single acyl groups such as butyryl or succinyl, or even long-chain fatty acyl groups (3). The relevance of this duality goes beyond sirtuins, as we know of just a handful of enzymes, most of them prokaryotic, that combine two activities in a single protein $(4,5)$.

By far, the best studied ADP-ribosyltransferase (ADPRT) is poly(ADP-ribose) polymerase 1 (PARP1), which is responsible for up to about $90 \%$ of the cellular poly-ADP-ribosylation (pADPR) generated by DNA damage and is a key pharmacological target for the treatment of DNA repair-deficient cancers. In contrast to the relatively well studied pADPR, the biological relevance of mADPR is more limited. $\mathrm{mADPR}$ has been shown to regulate a wide range of functions, from cellular stress response upon DNA damage or metabolic stress to infectious diseases and cancer (6). Reflecting their functional relevance and complexity, there are not only different classes of protein-binding modules (readers) that recognize ADPribosylation but also enzymes involved in the removal of ADP-ribose called ADP-ribosylhydrolases (erasers) (6). Among the readers, macrodomains are an ancient group of protein domains formed by 130 to 190 residues present from viruses to humans that bind mono(ADP-ribose), poly(ADP-ribose), and $\mathrm{NAD}^{+}$-derived metabolites. In humans, at least 12 proteins contain macrodomains belonging to four of the six known classes of macrodomains. One of the best studied macrodomain-containing proteins is the histone $\mathrm{H} 2 \mathrm{~A}$ variant macroH2A (mH2A). Of the three $\mathrm{mH} 2 \mathrm{~A}$ isoforms ( $\mathrm{mH} 2 \mathrm{~A} 1.1$, $\mathrm{mH} 2 \mathrm{~A} 1.2$, and $\mathrm{mH} 2 \mathrm{~A} 2$ ), only $\mathrm{mH} 2 \mathrm{~A} 1.1$ has the ability to bind ADPR (7). Moreover, mH2A1.1 can also bind free ADP-ribose molecules and the product of sirtuin deacetylation, $O$-acetyl-ADPribose (8), which suggests that $\mathrm{mH} 2 \mathrm{~A} 1.1$ may play a role as an 
energetic/metabolic sensor and could be functionally linked to the stress response role of sirtuins. $\mathrm{mH} 2 \mathrm{~A} 1.1$ has been implicated in gene repression and activation (9), in the latter case, especially after signal-induced gene activation through PARP1 inactivation.

Sirtuin-mediated ADP-ribosylation was described in yeast Sir2p before the discovery of the main deacetylation activity (10), and it was originally hypothesized that all sirtuins could potentially harbor mono-ADPRT (mADPRT) activity (11), although this activity is, in most cases, either orders of magnitudes slower than deacetylation or simply undetectable in in vitro studies (12). Among mammalian sirtuins (SirT1 to SirT7), only two-reported to be weak deacetylase enzymes in vitro $(13,14)$ - are known to ADP-ribosylate other protein targets. SirT4-dependent ADP-ribosylation of glutamate dehydrogenase represses its enzymatic activity by limiting the metabolism of glutamate and glutamine to produce adenosine 5 '-triphosphate (15). SirT6 can mediate the ADP-ribosylation of two targets: PARP1 under oxidative stress, thereby inducing double-strand break repair after DNA damage (16), and KRAB-associated protein-1 (KAP1), to promote long interspersed nuclear element-1 (LINE-1) silencing and genomic stability (17). SirT6 was also found to auto-ADP-ribosylate itself (18), although this activity has not been characterized. In vitro kinetic analysis suggests that the deacetylase activity of SirT6 with an acetylated $\mathrm{H} 3 \mathrm{~K} 9$ peptide is about 1000 times weaker than that of other sirtuins (14). Both enzymatic activities in SirT6 seem to involve specific residues within the same catalytic domain since specific mutations in this domain can selectively abrogate either mADPRT or deacetylase without altering the other activity (16). The coexistence of the two activities in the same protein is uncommon and raises many questions about the nature of this duality, the cross-talk between the two activities, and their specific contribution to the physiological role of sirtuins.

SirT7 is the closest sirtuin to SirT6 and probably one of the least studied. Its deficiency has been linked to genome instability and several pathologies, including cancer and aging $(19,20)$. SirT7 is involved in active ribosomal DNA (rDNA) transcription (21), DNA repair (20), metabolic homeostasis (22), and heterochromatin silencing and active transcriptional elongation through deacetylation of histone $\mathrm{H} 3$ lysine 18 (H3K18ac) and histone $\mathrm{H} 3$ lysine 36 (H3K36ac), respectively $(19,23)$. Recently, a direct link between SirT7, nuclear lamina, and LINE-1 has been described, suggesting a role for SirT7 in genome organization (24). SirT7 knockout (KO) mice show severe metabolic defects, including resistance to high-fat diet-induced fatty liver, obesity, glucose intolerance, and diminished levels of hepatic triglycerides and white adipocyte tissue $(25,26)$. In addition, these mice exhibit developmental defects and aging-related phenotypes such as perinatal lethality, degenerative heart hypertrophy, osteopenia, and kyphosis, and they die significantly younger than their wildtype $(W t)$ counterparts $(20,27)$. While there is a large body of evidence on sirtuin function and longevity, the mechanism by which SirT7 controls aging and the relationship between caloric restriction response and gene expression programs have not yet been adequately addressed.

Here, we describe how SirT7 harbors an auto-mADPRT activity at an alternative catalytic site in addition to its primary catalytic site, which is responsible for the deacetylation reaction. Auto-mADPRT of SirT7 confers its binding capacity on specific chromatin regions through the histone variant $\mathrm{mH} 2 \mathrm{~A} 1.1$, an interaction that is boosted under glucose starvation (GS), which promotes accumulation of mH2A1 in a SirT7-dependent manner. To advance our understanding of SirT7 function under energy-stress conditions, we sought evidence of the key role of the SirT7/mH2A axis in calorierestricted mice. This axis epigenetically regulates the expression of genes involved in differentiation, proliferation, metabolism, autophagy, and aging pathways. Our functional studies suggest a direct role for SirT7 in the control of the stress response through this newly found enzymatic activity.

\section{RESULTS}

\section{SirT7 is an mADPRT}

Several lines of evidence suggest that SirT7 may also harbor mADPRT activity. SirT7 and SirT6 are close homologs with a common origin in early eukaryotes. They consistently show a high level $(42 \%)$ of sequence identity and structure in their catalytic core domain. SirT7 shares a unique feature with SirT6 in the catalytic domain that is different from other mammalian sirtuins (Fig. 1A). Whereas there is a large helix bundle in the $\mathrm{NAD}^{+}$-binding Rossmann fold in the catalytic region of most sirtuins that connects this domain with a small $\mathrm{Zn}$-binding domain, this structure is missing from SirT6 and SirT7. This feature was thought to considerably reduce the flexibility of the structure, causing the low deacetylase activity detected in SirT6 (14). Consistent with this, the in vitro catalytic activity of SirT7 with H3K18ac is considerably weaker than that of other sirtuins such as SirT1 or SirT2 (23). Considering all this evidence, we aimed to determine whether SirT7 was also a dual sirtuin and whether it harbored an ADPRT activity. Our first approach was to use an in vitro ADP-ribosylation assay, using $\left[{ }^{32} \mathrm{P}\right]$-labeled $\mathrm{NAD}^{+}$ to determine whether SirT7 was able to transfer $\left[{ }^{32} \mathrm{P}\right]$-ADP-ribose to recombinant core histones. The ADPRT reactions of SirT7 on recombinant and native histones were very weak, but we detected a very efficient incorporation of $\left[{ }^{32} \mathrm{P}\right]$ in SirT7, strongly suggesting that SirT7 is itself a major target of this activity (Fig. $1 B$ and fig. S1A). The absence of a smear in the $\left[{ }^{32} \mathrm{P}\right]$ signal indicated that, as with SirT6, SirT7 harbors mADPRT activity (Fig. 1C). This auto-ADPRT activity was not altered by mutations previously described as abrogating SirT6 ADP-ribosylation activity toward PARP1 and KAP1 $(16,17)$. Mutation of SirT6 G60 to alanine or its equivalent in SirT7 S115 did not abrogate this auto-ADPRT activity. Moreover, the same result was obtained upon mutation of SirT6 S56 (fig. S1B), which was previously shown to eliminate both SirT6 activities, or of its equivalent in SirT7 S111 (Fig. 1D). In contrast to the close parallelism observed between SirT6 and SirT7 ADP-ribosylation activity, SirT7 S111 or S115 mutations did not abrogate the deacetylase activity (fig. S1C). This result, which was previously reported for S111 (28), suggests that the deacetylation active site of SirT6 and SirT7 may be not as equivalent as had been proposed previously. Nevertheless, our observations led us to consider the possibility that sirtuin auto-mADPRT activity is not associated with the previously described active site.

\section{SirT7 auto-mADPRT activity depends on the highly conserved residues, E185 and N189, present in the ELHGN motif}

To define this site, we compared all seven sirtuins at the structural level. In contrast to SirT1-5, the lack of the $\alpha$ helix bundle (in cyan; Fig. 1E, left) in SirT6 and SirT7 (Fig. 1E, right) induced a reorganization in the center of the structure that created a big cavity (Fig. 1E, red rectangle), located at the other side of the main catalytic site (in red). The cavity contained an ELHGN motif, conserved in the SirT6/SirT7 lineage, including the common SirT6/SirT7 ortholog Caenorhabditis elegans Sir-2.4 (Fig. 1F and fig. S1D). SirT7 H187, 
A

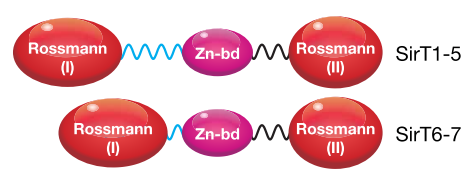

C

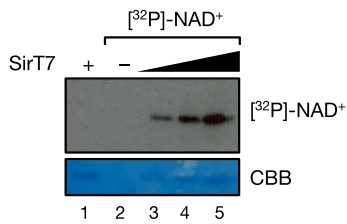

D
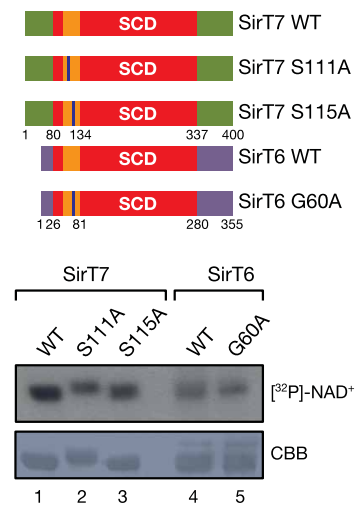

G

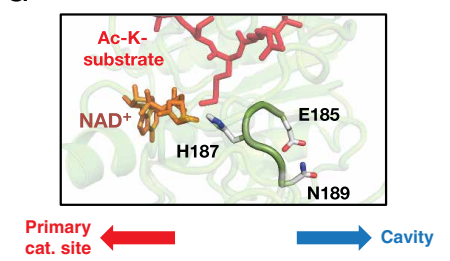

B

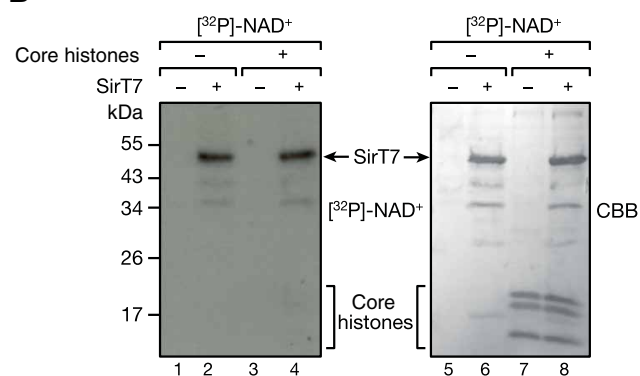

E
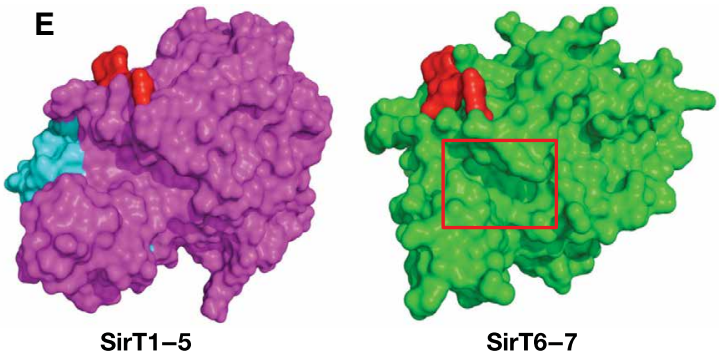

SirT6-7

F

$$
\begin{array}{ll}
\text { SGLPRTAISELHGNMYIEVCT } & \text { hsSirT7 } \\
\text { SGLPRTAISELHGNYIEVCT } & \text { mSirT7 } \\
\text { SGLPRNSLEIHGNMYEVCK } & \text { dmSirT7 } \\
\text { SGFPRDKLAELHGNMFVEECA } & \text { hsSirT6 } \\
\text { SGFPRDKLAELHGNMFVECP } & \text { mSirT6 } \\
\text { SGLDRYYSELHGIYIEQCK } & \text { dmSirT6 } \\
\text { VGIPVEDLIELGNLFEVCQ } & \text { ceSir- } 2.4 \\
\text { VAGIQR-IIQCHGSFATACL } & \text { hsSirT1 } \\
\text { AGLEQEDLVEAHGTFYTSHCV } & \text { hsSirT2 } \\
\text { AGS--RRLTELHGCMDRLCL } & \text { hsSirT4 }
\end{array}
$$
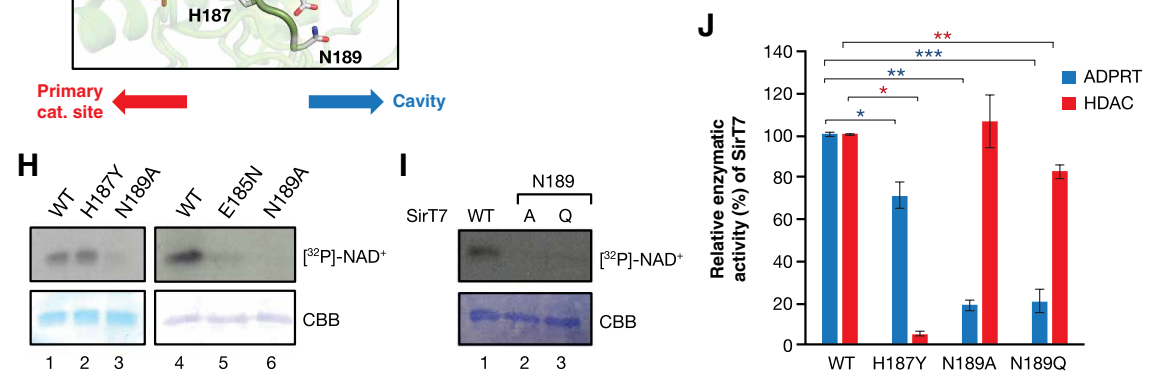

Fig. 1. SirT7 harbors an mADPRT activity that is catalyzed by an alternative domain. (A) Secondary structure of the conserved domain in mammalian sirtuins. Differences in $\alpha$ helix length between SirT1 to SirT5 and SirT6 to SirT17 are shown in blue. (B) In vitro ADPRT assay of SirT7 purified from human embryonic kidney (HEK) $293 \mathrm{~F}$ cells $\pm\left[{ }^{32} \mathrm{P}\right]-\mathrm{NAD}^{+}$with purified HeLa core histones. Autoradiography $\left.\left({ }^{32} \mathrm{P}\right]-\mathrm{NAD}^{+}\right)$and Coomassie blue staining are shown. CBB, Coomassie Brilliant Blue. (C) SirT7 titration in ADPRT assay as in (B). (D) Top: Schematic representation of SirT7 and SirT6 proteins tested. Bottom: ADPRT assay performed as in (B) and (C) with the indicated proteins. (E) Structural comparison of SirT1 to SirT5 (magenta) and SirT6 and SirT7 (green) conserved domains. The primary catalytic (cat.) site with a bound acetylated peptide is shown in red, while the $\alpha$ helix bundle missing in SirT6 and SirT7 is shown in cyan. Loss of this region induces the formation of a cavity in SirT6 and SirT7 (red square). (F) Alignment of the sequence present in the cavity in the SirT6 and SirT7 lineage. E185, H187, and N189 are shown in green, blue, and red, respectively. (G) Molecular model of the SirT7 catalytic domain based on the crystal structure of SirT6. $\mathrm{H} 187$ is oriented toward the NAD molecule (orange) and the acetylated substrate (red). E185 and N189 are located in the same loop as H187 although pointing in the opposite direction. (H and I) In vitro auto-ADPRT assay of SirT7 proteins purified from HEK293F cells. (J) Deacetylation and ADPRT activity of the indicated proteins. The mADPRT and deacetylation activities were quantified from assays as in (H) and as in fig. $\mathrm{S} 1 \mathrm{H}$, respectively. For the deacetylation reaction, the indicated proteins were incubated with hyperacetylated core histones purified from HeLa cells and the deacetylation of H3K18ac was monitored by Western blot. Quantifications of both activities $(n=3)$ are shown relative to WT SirT7 activity $\left({ }^{*} P<0.05\right.$, ${ }^{* *} P<0.01$, and $\left.{ }^{* * *} P<0.005\right)$.

present in the motif, is a key conserved residue among sirtuins involved in the recognition of acetylated substrate in the deacetylation activity. While $\mathrm{H} 187$ was oriented toward the $\mathrm{NAD}^{+}$-binding pocket and the main catalytic site, the two flanking residues, E185 and N189, faced in the opposite direction, toward the surface of the cavity (Fig. $1 \mathrm{G}$ and fig. S1, E and F), forming a loop sustained by the inter- action of both residues through their side chain. We paid particular attention to these two conserved residues because of their possible involvement in the ADP-ribosylation reaction: E185 is the only residue in this motif that could initiate the reaction, whereas N189, the only residue in the motif conserved exclusively in the whole SirT6/SirT7 lineage (Fig. 1F), could act as the first acceptor of the ADP-ribosyl moiety. This possibility 
was confirmed with the finding that the E185N and N189A mutations both abrogated SirT7 auto-mADPRT activity (Fig. 1H).

A highly conservative mutation to glutamine (N189Q) had the same effect, suggesting that the lack of activity of the N189 mutant was not due to an indirect structural effect (Fig. 1I). The fact that N189Q is structurally very similar to the WT protein (fig. S1G) but cannot replace glutamine as acceptor of ADP-ribose suggests that N189 may play an essential role in the mechanism of ADP-ribosylation. N189 mutants were defective in ADPRT activity but still featured deacetylase activity, while the H187Y mutant, just two residues away, had the completely opposite pattern (Fig. 1J and fig. S1H). Considered together, these observations indicate that SirT7 automADPRTion is catalyzed at a conserved alternative secondary active site located in a previously uncharacterized domain.

\section{N189-dependent auto-mADPRT involves several residues distributed around the SirT7 surface and regulates SirT7 genomic distribution}

We also confirmed the conserved role of N189 since the equivalent mutation in SirT6 N135 also abrogated SirT6 mADPRT activity (Fig. 2A) but did not eliminate its deacetylase activity toward H3K18ac (Fig. 2B and fig. S2A). There were two further lines of evidence of the importance of N189: First, the N189 mutant was significantly less ADP-ribosylated in vivo than was WT SirT7 (Fig. 2C); second, mass spectrometry (MS) analysis of SirT7 auto-mADPRTion identified eight ADP-ribosylated peptides distributed across the entire surface of the SirT7 protein (Fig. 2, D and E; fig. S2, B and C; and table S1). All but one of them were undetected in the N189 mutant (Fig. 2E, in magenta), confirming a key role for N189 in SirT7 ADPRT. Together, these observations strongly suggest that SirT7 auto-mADPRTion is catalyzed at an alternative secondary active site.

Taking advantage of our ability to specifically inhibit each of the two SirT7 enzymatic activities, we next studied the impact of these mutations on SirT7 distribution. Our first step was to study immunofluorescence (IF) signal of WT, H187Y, N189A, and N189Q in SirT7 ${ }^{-1-}$ mouse embryonic fibroblasts (MEFs). We observed that while H187Y had an identical distribution to WT SirT7, whereby it was enriched in the nucleolus, the patterns of localization of N189A and N189Q were radically different. Both N189 mutants showed a more dispersed pattern of distribution that resulted in a loss of nucleolar enrichment in around $80 \%$ of the observed cells (Fig. 2, F and G). Although we observed a mild decrease in the expression of $\mathrm{N} 189$ mutants compared to SirT7 WT and H187Y, these differences could not account for the marked changes observed in the distribution of these proteins (Fig. 2H). The altered distribution of these mutants was confirmed by biochemical fractionation of cells expressing SirT7-flagged mutants into cytoplasm, soluble nuclear fraction (nucleoplasm), and chromatin-insoluble fraction (chromatin). As in the IF studies, H187Y behaved similarly to WT and was present in similar levels in the nucleoplasm. By contrast, N189A-Q mutants were significantly enriched in the chromatin fractions and depleted in the nucleoplasm (Fig. 2H). Overall, our results suggest that, in contrast to SirT7 deacetylase activity, mADPRT activity, and, in particular, N189, plays a key role in both SirT7 genomic localization and chromatin-binding dynamics.

\section{SirT7 interacts with the ADP-ribose binding protein mH2A1.1 upon GS}

We tried to identify SirT7 binding partners to understand the functional link between SirT7 and ADP-ribosylation. We performed
SirT7 affinity purification in human embryonic kidney (HEK) 293F cells and identified potential SirT7 interactors by MS analysis. We identified the histone variant $\mathrm{mH} 2 \mathrm{~A} 1$ from among the candidates (Fig. 3A and table S2). mH2A1.1 was the only one of the three $\mathrm{mH} 2 \mathrm{~A}$ isoforms that specifically binds ADP-ribose through its macrodomain (Fig. 3B) (7) and interacted specifically with SirT7 (Fig. 3C). We next examined whether the SirT7-mH2A1.1 interaction depends on SirT7 ADP-ribosylation by incubating bacterially expressed recombinant SirT7 (rSirT7) WT or N189A in the presence or absence of $\mathrm{NAD}^{+}$and dialyzing the reaction to remove all remnant $\mathrm{NAD}^{+}$molecules. We tested its ability to specifically pull down mH2A1.1 from HEK293F nuclear extracts under stringent conditions (Fig. 3D). We found that only rSirT7 WT previously incubated with NAD ${ }^{+}$ was able to interact with WT mH2A1.1 under these conditions (Fig. 3E). Consistently, while the ADPRT-defective mutant N189A was not able to interact in any of these conditions (Fig. 3D), the deacetylase mutant H187Y behaved similarly as SirT7 WT (fig. S3A). As further evidence that SirT7 auto-mADPRT mediates this binding, no interaction was detected under these conditions when we used mH2A1.1 G224E (Fig. 3E, lanes 5 and 6), a mutant unable to bind specifically to ADP-ribose (7). This is compelling evidence that SirT7 auto-mADPRT mediates this binding.

The in vivo interaction between SirT7 and mH2A1.1 was specific but weak. Considering the direct link between sirtuins and the stress response, we investigated whether the SirT7-mH2A1.1 interaction is boosted under specific stress conditions, such as ultraviolet (UV) or ionizing irradiation (IR), oxidative stress $\left(\mathrm{H}_{2} \mathrm{O}_{2}\right)$, or GS. Under highly stringent conditions, there was a significant increase in the interaction between the two factors, specifically upon GS (Fig. 3F), which was consistent with the established link between both proteins and glucose homeostasis $(8,25)$. MS analysis of SirT7-FLAG purified under these stress conditions identified SirT7 ADP-ribosylation under UV, oxidative, and GS conditions but not in the absence of stress (C) or under IR [Fig. 3F (bottom), fig. S3, and table S1]. The ADP-ribosylation detected under GS and IR had been observed to be N189-dependent in our previous experiment (Fig. 2D). This discrepancy between the stress-induced SirT7 ADP-ribosylation and binding to mH2A1.1 suggested that while ADP-ribosylation is essential for binding to $\mathrm{mH} 2 \mathrm{~A} 1.1$, other GS-specific signaling may be important to drive the interaction. In contrast to normal culture conditions [no treatment (NT)], GS consistently induced coelution of both factors in high molecular weight fractions (around $1 \mathrm{MDa}$ ) of gel filtration chromatography analysis (Fig. 3G). This observation suggests that both factors may be part of a multiprotein complex formed under these conditions. As further evidence of this link, SirT7 was able to immunoprecipitate endogenous $\mathrm{mH} 2 \mathrm{~A} 1.1$ specifically under GS (Fig. 3H, lanes 3 and 4), an interaction that was abrogated by $\mathrm{N} 189$ mutation (Fig. $3 \mathrm{H}$, lanes 4 to 6 ).

\section{SirT7 binds to distal intergenic regions upon GS in an mH2A1-dependent manner}

While the levels of total $\mathrm{mH} 2 \mathrm{~A} 1$ and $\mathrm{mH} 2 \mathrm{~A} 1.2$ decreased upon GS, we observed a consistently specific increase of $\mathrm{mH} 2 \mathrm{~A} 1.1$ under the same conditions, suggesting a direct role for this isoform in the GS response (Fig. 4A). Consistently, biochemical fractionation analysis of SirT7 also showed a progressive accumulation of SirT7 in chromatin fractions upon GS, peaking at 40 hours of treatment (Fig. 4B). We chose this specific timing, as we determined experimentally that the GS-dependent enrichment of SirT7 was detectable from 24 hours of 
A

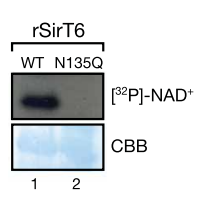

B

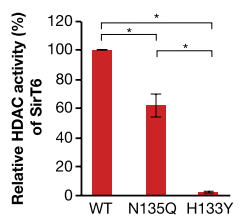

C

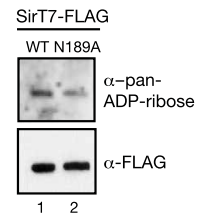

E

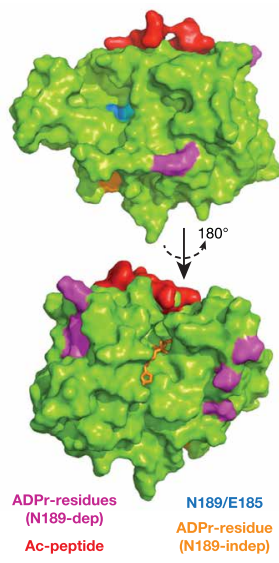

H

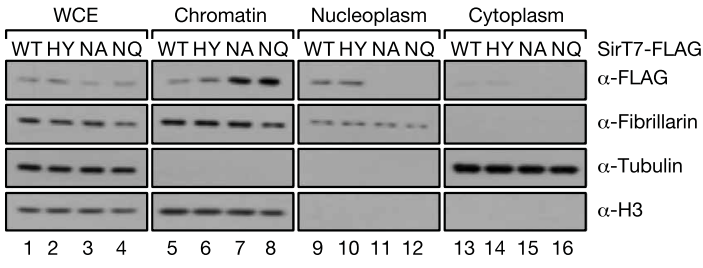

F
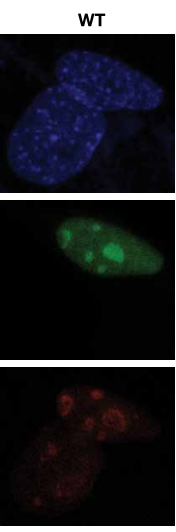

D
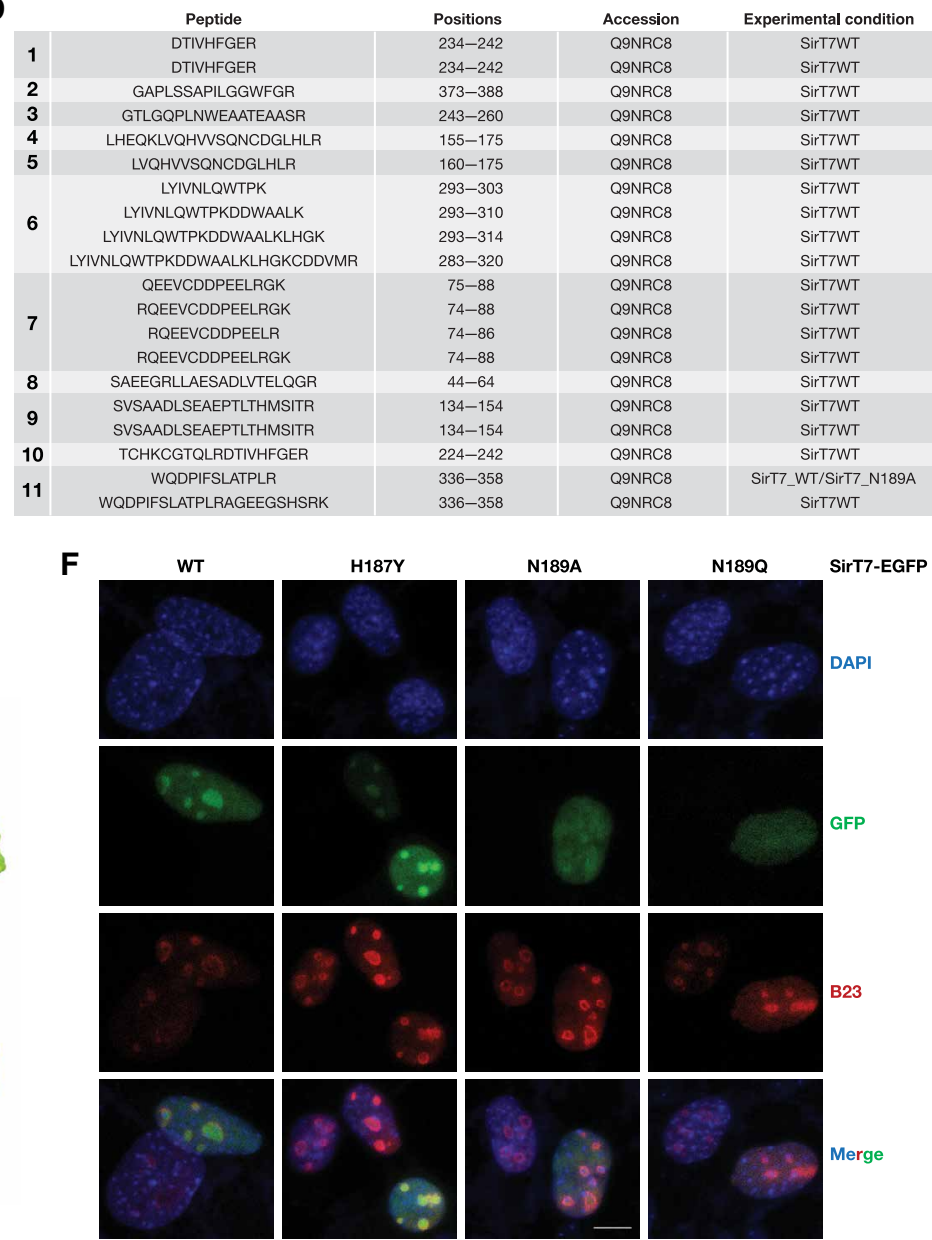

G

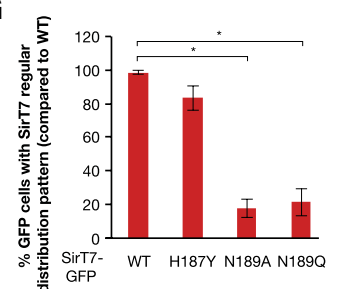

Fig. 2. SirT7 N189-dependent auto-ADP-ribosylation regulates SirT7 distribution and chromatin-binding dynamics. (A) In vitro auto-mADPRT assay as in Fig. 1 with bacterially expressed rSirT6 WT or N135Q. (B) Deacetylation reaction with the recombinant bacterial SirT6 WT, N135Q, and H133Y incubated with recombinant mononucleosomes acetylated in H3K18ac ( $\left.{ }^{*} P<0.05\right)$. Quantification of three experiments similar to the one shown in fig. S2A. The results are represented relative to WT SirT7 activity (100\%). (C) ADP-ribosylation levels of SirT7 WT or N189A expressed in HEK293F cells monitored by far Western blot with anti-pan-ADP-ribose binding reagent. HDAC, histone deacetylase. (D) Analysis of SirT7 auto-mADPRTion identified by MS. ADP-ribosylated peptides identified in SIRT7 WT or N189A after incubation with $\mathrm{NAD}^{+}$were analyzed using high-energy collisional dissociation (HCD) and electron-transfer/higher-energy collision dissociation fragmentation methods. The most probable ADP-ribosylated sites are highlighted in red, as the modification cannot be localized with 100\% confidence. Further information is included in table S1 and in Materials and Methods. (E) Structural model of the SirT7 catalytic domain indicating localization of the ADP-ribosylated peptides identified in (C). The ADP-ribosylation N189dependent (magenta) and N189-independent (orange) residues, the N189/E185 cavity (blue), and the primary catalytic site bound to acetylated peptide (red) are shown. (F) IF assay of the indicated green fluorescent protein (GFP)-tagged SIRT7 proteins expressed in SirT7 $7^{-1-}$ MEFs. Nucleophosmin (B23) was included as a nucleolar marker. DAPI, 4',6-diamidino-2-phenylindole. A representative image of the experiment is shown. Scale bar, $5 \mu \mathrm{m}$. (G) Quantification of IF experiment in (F), indicating the percentage of GFP-positive cells with a regular nucleolar distribution ( $\left.{ }^{*} P<0.05\right)$. (H) Cellular distribution of SirT7 WT, H187Y (HY), N189A (NA), and N189Q (NQ) in whole-cell extract (WCE), cytoplasm, nucleoplasm, and chromatin in NIH3T3 cells. Controls for the nuclear fraction (fibrillarin), cytoplasm (tubulin), and chromatin (histone H3) are also shown.

GS and that 40 hours was the maximum safe time of GS treatment before entering apoptosis. To understand the functional relationship between the two factors under GS, we next studied the distribution of SirT7 and mH2A1 under NT and GS conditions in MEFs.
Supporting the biochemical fraction results, SirT7 chromatin immunoprecipitation sequencing (ChIP-seq) analysis showed that GS boosted SirT7 localization to chromatin (Fig. 4C). In the case of mH2A1, we observed a SirT7-dependent global enrichment of 
A

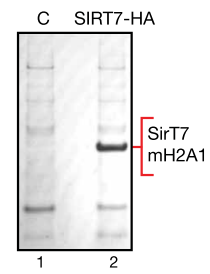

D

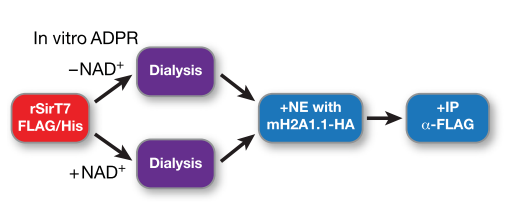

E AISSYFVSTMSSSIK AASADSTTEGTPADGFTVLSTK AGVIFPVGR GVTIASGGVLPNIHPELLAK SLFLGQK NGPLEVAGAAVSAGHGLPAK LEAIITPPPAKK QTAAQLILK TPADGFTVLSTK NCLALADDKKLK

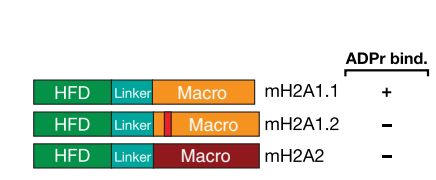

C

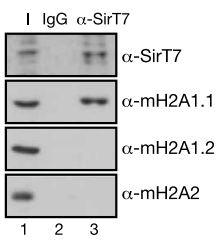

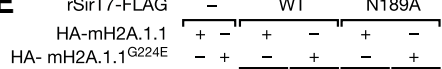

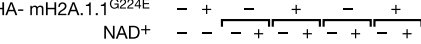

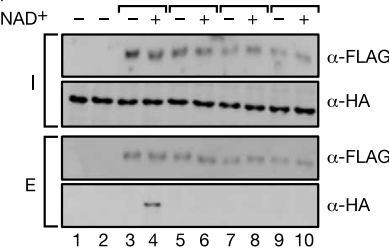

$\mathbf{F}$

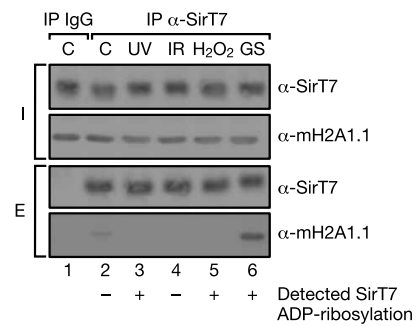

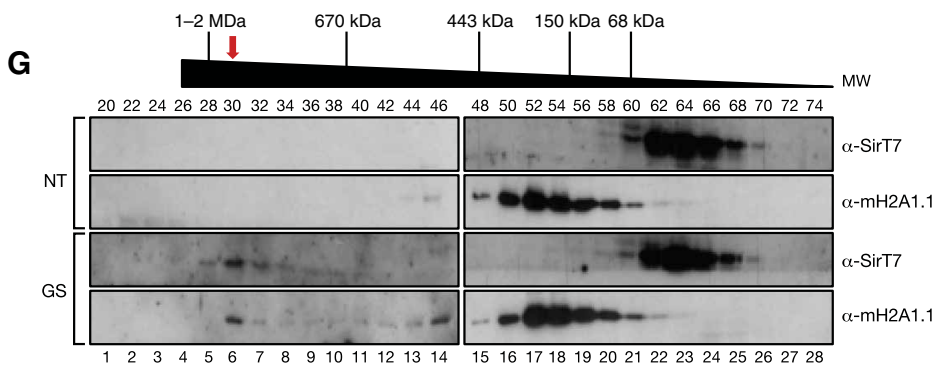

H

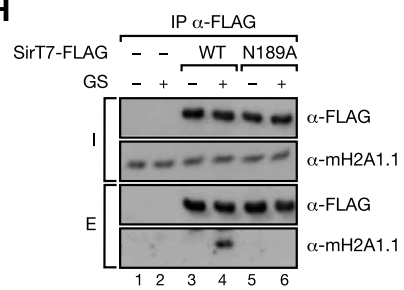

Fig. 3. SirT7 interacts with mH2A1.1 upon GS in an ADP-ribosylation-dependent manner. (A) Affinity purification of SirT7-binding factors identified the histone H2A variant $\mathrm{mH} 2 \mathrm{~A} 1$ by MS analysis (table $\mathrm{S} 2$ ). $\mathrm{HA}$, hemagglutinin. (B) Schematic representation of the three $\mathrm{mH} 2 \mathrm{~A}$ isoforms, of which, only $\mathrm{mH} 2 \mathrm{~A} 1.1$ binds to ADP-ribose (7). (C) Endogenous SirT7 specifically immunoprecipitates mH2A1.1 in HEK293F cells. IgG, immunoglobulin G. (D and E) mH2A1.1 recognizes and binds ADP-ribosylated SirT7. Immunoprecipitation (IP) of bacterially expressed rSirT7 WT or N189A preincubated \pm NAD ${ }^{+}$and added to nuclear extracts of HEK293F SirT7 KO cells (CRISPR-Cas9mediated KO of SIRT7) expressing mH2A1.1 WT or G224E, a mutant deficient in ADP-ribose binding. Inputs (I) and elutions (E) are shown. A similar experiment with H187Y is shown in fig. S3A. (F) Top: Interaction between endogenous SirT7 and mH2A1.1 under high-stringency conditions upon different types of stress in HEK293 cells. C, untreated; IR, 7-gray ionizing irradiation; $\mathrm{H}_{2} \mathrm{O}_{2}$, oxidative stress. Western blot of the input and elution of immunoprecipitation experiments with anti-SirT7 antibody under these conditions. Bottom: Summary of the ADP-ribosylation events detected by MS in SirT7-FLAG expressed in the same cells and purified under the same stress conditions. Further details are shown in table in fig. S3B and in table S1. (G) Superose 6 gel filtration chromatography of nuclear endogenous proteins from nuclear extracts of HEK293F cells under normal cell growth (NT) or upon GS. Fraction numbers and approximate molecular weights (MW) are indicated. Western blot of SirT7 and mH2A1.1 are shown. (H) High-stringency immunoprecipitation of endogenous mH2A1.1 by WT or N189A SirT7 in HEK293F cells treated under normal conditions or under GS. Inputs (I) and elutions (E) are shown.

mH2A1 in Wt cells upon GS compared with NT conditions since this effect was abrogated in SirT7-deficient MEFs (Fig. 4D). In addition, $72.4 \%$ (1711 of 2638) of SirT7-associated genes were also enriched by mH2A1. SirT7 accumulated mostly in intergenic regions [transcription start site (TSS) \pm 50 to $500 \mathrm{~kb}$ ] rather than in proximal promoters (Fig. 4E). Genomic Regions Enrichment of Annotations Tool (GREAT) analysis associated these regions with specific genes (Fig. $4 \mathrm{~F}$ and fig. S4), suggesting that the sequences correspond to gene regulatory regions. The average distance from these sites to the associated genes ranges between 50 and $500 \mathrm{~kb}$ upstream or downstream of the coding region. Many of the SirT7-associated genes mapped by GREAT were associated by Kyoto Encyclopedia of Genes and Genomes (KEGG) analysis with cell signaling, the majority of which directly or indirectly participate in regulating metabolic homeostasis (Fig. 4F).

Confirming our previous results, the recruitment of SirT7 to these regions upon GS was mH2A1 dependent since its short hairpin RNA (shRNA)-driven down-regulation abrogated SirT7 enrichment (Fig. 4G). These results indicate that, upon GS, mH2A1.1 recruits
SirT7 to the intergenic regions and suggest that SirT7 has a direct role in auto-mADPRTion.

\section{The SirT7/mH2A1 regulatory axis regulates the expression of a subset of genes upon GS}

To clarify the mode of action of SirT7 in the genes regulated by SirT7/mH2A1 when they are stressed, we performed RNA sequencing (RNA-seq) in Wt and Sirt $7^{-1-}$ MEFs under NT and GS as for the ChIP-seq analyses. Of the $1711 \mathrm{mH} 2 \mathrm{~A}$-containing regions occupied by SirT7, GS induced the up-regulation and down-regulation of 257 and 427 nearby genes, respectively, in Wt MEFs (Fig. 5, A and B). This up- or down-regulation upon GS was SirT7 dependent in the case of 143 and 277 genes, respectively (Fig. 5B). There was an increase in the levels of mH2A1 around these genes upon GS that was abrogated in SirT7-deficient cells (Fig. 5C and fig. S5). Among these genes, we identified some that encoded growth factors, signaling transduction mediators involved in metabolism and differentiation, hormone receptors, enzymes, transcription factors, extracellular factors, and cytokines. Many of these genes are directly or indirectly 

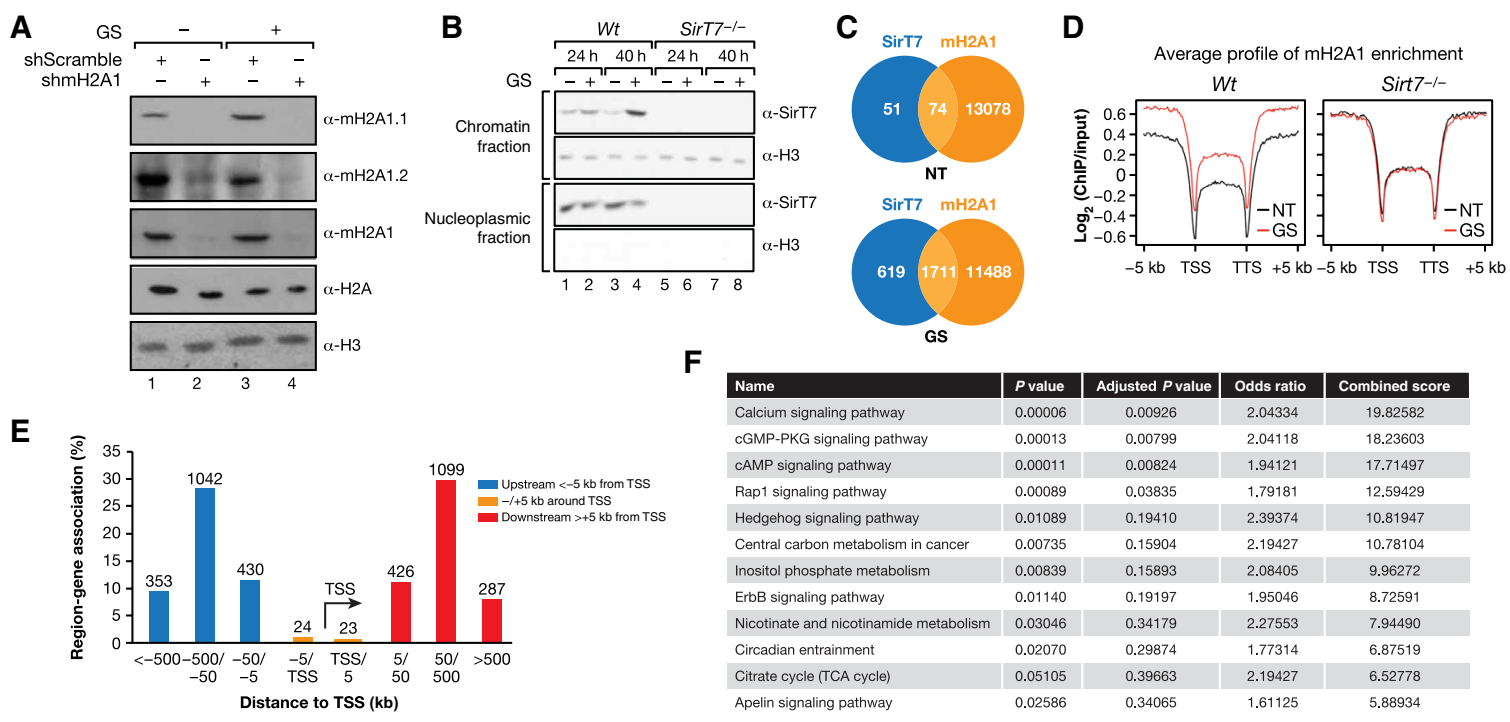

G
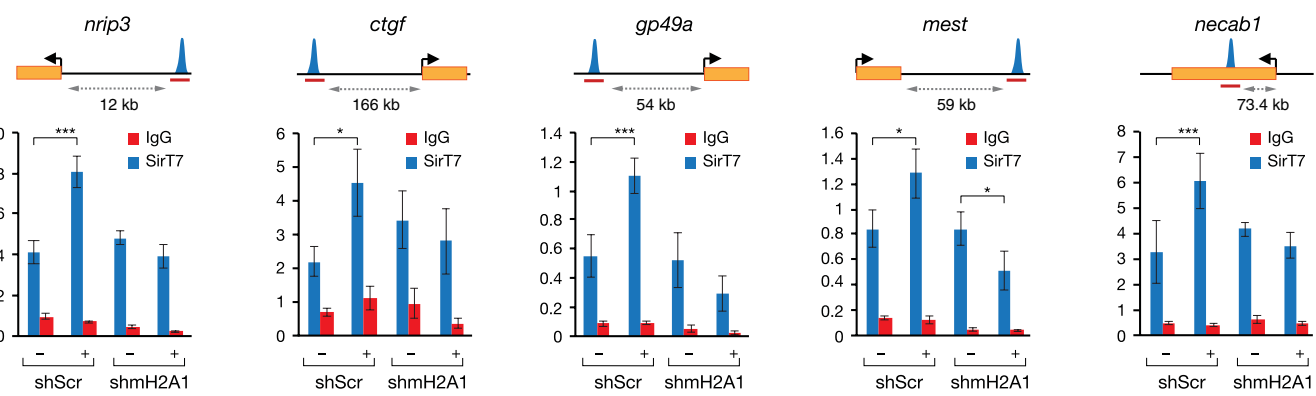

Fig. 4. $\mathrm{mH} 2 \mathrm{~A} 1$ recruits SirT7 to distal intergenic regions associated with metabolic genes upon GS. (A) Levels of three $\mathrm{mH} 2 \mathrm{~A}$ isoforms in WCE of NIH3T3 cells transfected with scramble shRNA or mH2A1 shRNA and cultured under normal or GS conditions. (B) Levels of endogenous SirT7 and histone H3 in chromatin and nucleoplasm fractions purified from Wt and Sirt $7^{-1-}$ MEFs cultured under normal or GS conditions during the indicated times. (C) Venn diagrams showing the intersection of SIRT7associated genes with $\mathrm{mH} 2 \mathrm{~A} 1$-enriched genes in Wt cells under NT (top) or GS (bottom). SirT7-associated and mH2A1-enriched genes were derived from GREAT analysis (fig. S3B and Materials and Methods). (D) Average enrichment of $\mathrm{mH} 2 \mathrm{~A} 1$ at all genes in Wt (left) or SirT7 $7^{-1}$ MEF (right) cells under NT (black) or GS (red) conditions. Data are expressed as the $\log _{2}$ ratio of reads per kilobase of transcript per million mapped reads-normalized ChIP/input signals. (E) Distribution of sites occupied by SirT7 upon GS around the TSS by GREAT analysis. The values for each bin from the TSS are shown above each bar (TSS: $-5 \mathrm{~kb}, 5$ to $50 \mathrm{~kb}, 50$ to $500 \mathrm{~kb}$, and >500 kb). (F) KEGG cell signaling pathways for SirT7-associated genes mapped by GREAT analysis under GS in MEF cells. The signaling pathways were ranked by their combined score provided by Enrichr analysis. CGMP-PKG, guanosine 3',5'-monophosphate-protein kinase G; cAMP, cyclic adenosine 3',5'-monophosphate; TCA, tricarboxylic acid. (G) SirT7 ChIP-qPCR (quantitative polymerase chain reaction) analysis of SirT7 binding sites associated with $\mathrm{mH} 2 \mathrm{~A} 1$ at distal regions upon shRNA-mediated down-regulation of $\mathrm{mH} 2 \mathrm{~A} 1 \mathrm{under}$ normal and GS conditions in NIH3T3 cells. The amplified regions (red) and their distance to each gene are indicated in the upper part of each graph. Each SirT7 ChIP was normalized with respect to its own input. SEM from $n=4$. Two-tailed $t$ test $\left({ }^{*} P<0.05\right.$ and $\left.{ }^{* * *} P<0.005\right)$.

associated with the second messenger regulatory system, including the $\mathrm{Ca}^{+2}$-dependent pathways and the adenosine $3^{\prime}, 5^{\prime}$-monophosphate (cAMP)/adenylate cyclase or guanosine $3^{\prime}, 5^{\prime}$-monophosphate (cGMP)dependent protein kinase signaling pathways. We also identified many factors associated with heterotrimeric GTP-binding protein (G protein) signaling, such as the $G$ protein-coupled receptor signaling pathways, some of which have been linked to regulation of cAMP signaling in neural systems. Genes down-regulated upon GS were involved in cAMP signaling pathway factors, proliferation, and cell differentiation, while up-regulated genes were mainly associated with cGMP-dependent protein kinase and Rap1 signaling, metabolism, and, to a much lesser extent, cAMP/adenylate cyclase signaling (fig. S6A). Among these up- or down-regulated factors that we identified are, for instance, the connective tissue growth factor (CTGF), the adenylate cyclase regulator adrenoceptor alpha 2A (ADRA2A), the guanylatebinding protein 6 (GBP6), the histone deacetylase 9 (HDAC9), the calcium-binding signaling protein $\mathrm{N}$-terminal EF-hand calcium binding protein 1 (NECAB1), and the transcription factors forkhead box protein A2 (FOXA2) and myocyte-specific enhancer factor 2C (MEF2C). CTGF was particularly interesting, given its key role in differentiation, proliferation, glucose homeostasis, and insulin-like growth factor 1 (IGF-1) and transforming growth factor- $\beta$ signaling and its link to calorie restriction (CR) and aging (29).

Reconstitution experiments with SirT7 WT, H187Y, and N189A in Sirt $7^{-/-}$MEFs revealed that both enzymatic activities of SirT7 are required to produce the GS-associated expression profile of the tested genes, as well as the associated enrichment of $\mathrm{mH} 2 \mathrm{~A}$ detected in these genes upon GS (Fig. 5D). Consistent with a direct role of SirT7 in these genes through binding to the distal regulatory regions, we also observed SirT7-dependent H3K36 deacetylation in these distal sequences, which was dependent on both catalytic activities (fig. S6B). These lines of evidence suggest a link between this deacetylation event and $\mathrm{mH} 2 \mathrm{~A}$ gene enrichment. As $\mathrm{mH} 2 \mathrm{~A} 1$ was previously associated with $\mathrm{H} 3 \mathrm{~K} 27 \mathrm{me} 3$ (30), we also analyzed the 
A

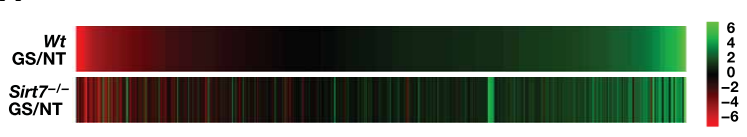

B

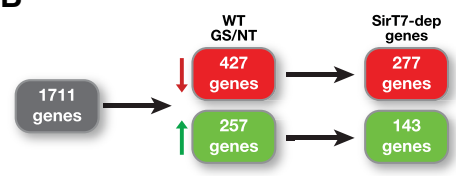

C
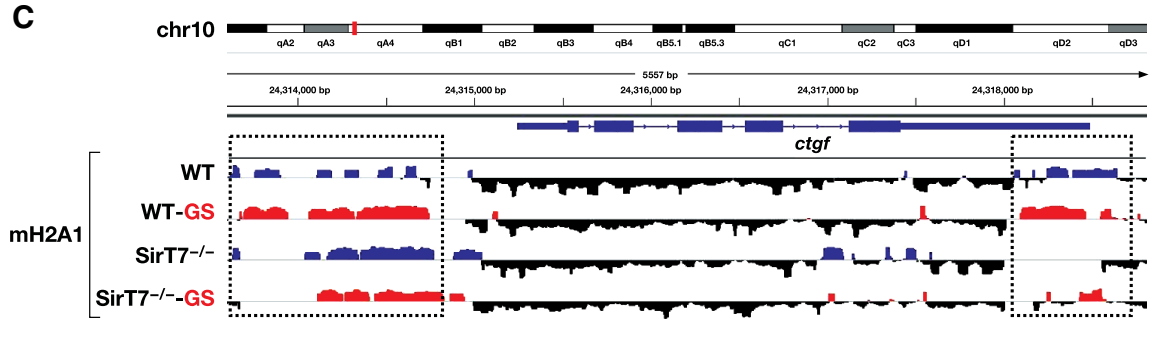

D
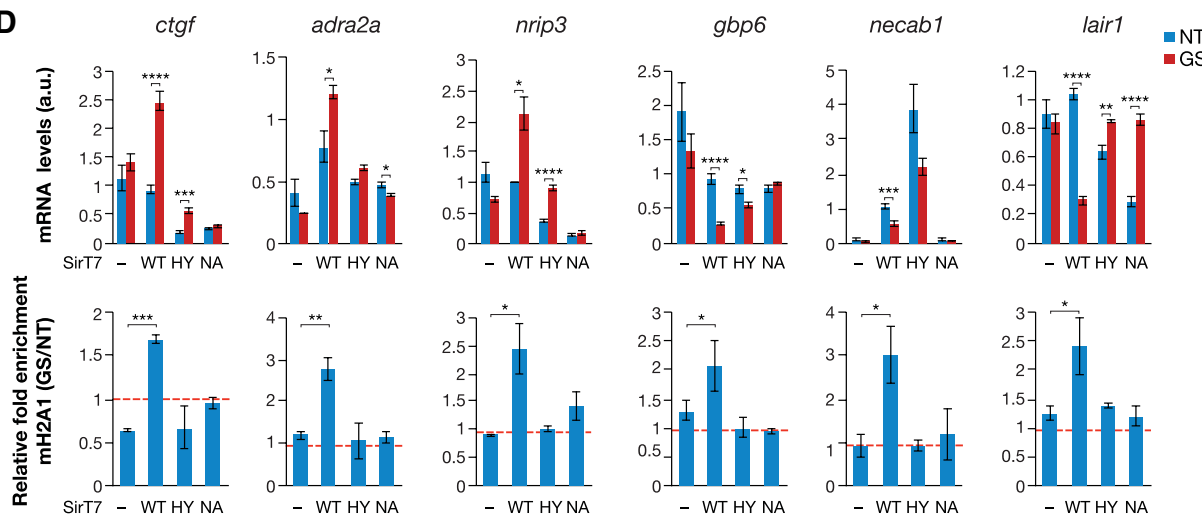

E
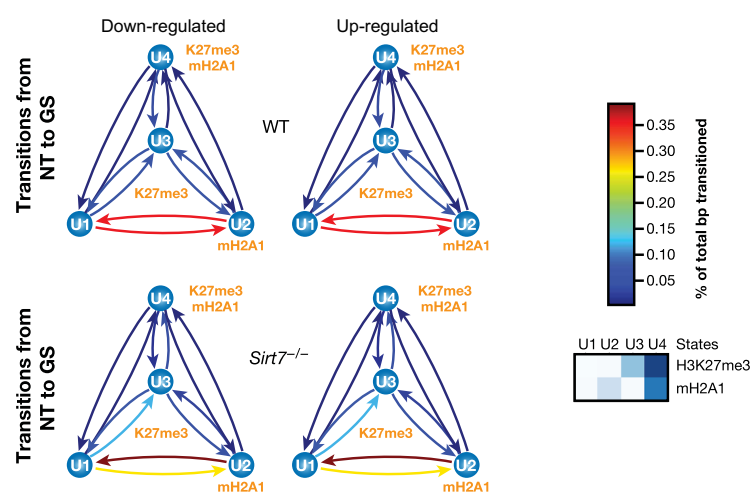

Fig. 5. SirT7 dual activity promotes $\mathrm{mH} 2 \mathrm{~A} 1$ enrichment in the promoters of associated genes, resulting in transcription up-regulation or down-regulation. (A) Heat map showing RNA expression changes relative to NT (as $\log _{2}$ magnitude of difference between GS and NT) conditions in Wt and SirT7-deficient MEFs. (B) Pipeline applied to RNA-seq data to filter genes associated with SirT7/mH2A1 in Wt and Sirt ${ }^{-1-}$ MEF cells treated under GS or NT. The analysis was restricted to genes that (i) were associated with SirT7 via GREAT and were $\mathrm{mH} 2 \mathrm{~A} 1$-enriched, (ii) showed a $\log _{2}$ fold change (FC) of expression between WT-GS and WT-NT $>0.6$, and (iii) showed a difference between WT and KO $\log _{2}$ FC (GS versus NT) of $>0.3$ (table S3). (C) $\mathrm{mH} 2 \mathrm{~A} 1$ ChIP-seq signals across ctgf, a gene differentially enriched in $\mathrm{mH} 2 \mathrm{~A} 1 \mathrm{upon}$ GS in $W t \mathrm{MEFs}$ compared with NT. (D) Top: Real-time qPCR (RT-qPCR) analysis of genes regulated by SirT7 upon GS and NT. The expression of SirT7 in SirT7 ${ }^{1-}$ MEFs was rescued by retroviralmediated gene transfer of SirT7 WT, H187Y(HY), N189A(NA), and empty vector (-). SEM from $n=4$. Two-tailed $t$ tests $\left({ }^{*} P<0.05,{ }^{* *} P<0.01,{ }^{* * *} P<0.005\right.$, and $\left.{ }^{* * * *} P<0.001\right)$. Bottom: Relative $\mathrm{mH} 2 \mathrm{~A} 1$ enrichment (GS versus NT) by ChIP-qPCR analysis at specific regions around the TSS of the indicated genes [ $g b p 6,-6 \mathrm{~kb} ;$ necab1, $-3 \mathrm{~kb}$; lair1, $-27.5 \mathrm{~kb}$; ctgf, +600 base pairs (bp); adra2a, $-5 \mathrm{~kb}$; and nrip $3,+7 \mathrm{~kb}$. SEM from $n=3$. One-way analysis of variance (ANOVA) $\left({ }^{*} P<0.05\right.$, ${ }^{* *} P<0.01$, and $\left.{ }^{* * *} P<0.005\right)$. a.u., arbitrary units. (E) Chromatin state transitions induced by GS in Wt and SirT7-deficient cells. The colors of the arrows indicate the frequency (\%) of the transition as stated in the color scale (right). Bottom right: State map illustrating the specific combination of $\mathrm{mH} 2 \mathrm{~A} 1$ and/or $\mathrm{H} 3 \mathrm{~K} 27 \mathrm{me} 3$ in the four chromatin states defined in the analysis. $\mathrm{U} 1$, without $\mathrm{H} 3 \mathrm{~K} 27 \mathrm{me} 3$ or $\mathrm{mH} 2 \mathrm{~A} 1$; U2, $\mathrm{mH} 2 \mathrm{~A} 1$; U3, H3K27me3; U4, enriched by H3K27me3 and mH2A1.

correlation between this mark and $\mathrm{mH} 2 \mathrm{~A} / \mathrm{SirT7}$ through a global unbiased analysis of chromatin state changes from NT to GS conditions in $W t$ and Sirt $7^{-1-}$ MEFs. We defined four states (U1 to U4), depending on the appearance or disappearance of $\mathrm{mH} 2 \mathrm{~A}$ and/or
H3K27me3 in these sequences upon GS. We observed a positive effect of SirT7 on mH2A recruitment upon GS in up-regulated and down-regulated genes when $\mathrm{H} 3 \mathrm{~K} 27 \mathrm{me} 3$ was not present. In contrast, when $\mathrm{mH} 2 \mathrm{~A} 1$ and H3K27me3 were both present, SirT7 had the 
opposite effect on mH2A enrichment (Fig. 5E). These results suggest an antagonistic interplay between SirT7 and H3K27me3 in determining $\mathrm{mH} 2 \mathrm{~A}$ enrichment upon metabolic stress (Fig. 5E).

\section{SirT7 dependence on the expression of these genes is recapitulated in in vivo mouse models of CR}

To confirm the contribution of SirT7 to GS response in vivo, we generated a CR model in $W t$ and Sirt $7^{-1-}$ mice (Fig. 6A) and we performed several correlative analyses in the livers of these mice. Several evidences supported our previous studies with GS. First, we studied the expression of the SirT7/mH2A-associated genes that we had identified earlier (Figs. 4 and 5). Consistently, the SirT7-dependent expression profile of these genes under CR versus ad libitum (AL) fully replicated our results in cell culture with GS versus NT (Fig. 6B). Second, as in the case of GS, SirT7 was enriched in chromatin of these livers upon CR, in part, because of an up-regulation of the levels of SirT7 under these conditions (Fig. 6C). Third, we demonstrated CR-specific interaction between endogenous SirT7 and $\mathrm{mH} 2 \mathrm{~A} 1.1$ in these livers, further supporting the functional parallelism between CR and GS (Fig. 6D). Fourth, trying to understand the functional implications of the SirT7-mH2A1 axis under CR conditions, we studied the status of autophagy in these samples, given the direct involvement of cAMP, cGMP, and $\mathrm{Ca}^{+2}$-dependent signaling in the control of autophagy and its established implication in the response to CR and aging (31-33). For that purpose, we analyzed the levels of a well-established marker of autophagy, the formation of the form II of microtubule-associated protein light chain 3 (LCIII-2). The rate of formation of LCIII-2 directly indicates the rate of autophagosome formation and activity. As expected, in $W t$ animals, we observed an increase of LCIII-2 under CR (Fig. 6E), in agreement with an increase in autophagy as a response to $\mathrm{CR}$ as has been described (33). In contrast, in SirT7-deficient mice, while we observed increased rates of autophagy in AL conditions, these decreased significantly compared to CR in $W t$ animals (Fig. 6E). Supporting a role for SirT7 in CR-dependent autophagy, we observed a similar pattern in Beclin-1 (Fig. 6F), another key marker of autophagy involved in the formation of autophagosomes (33). These observations suggest that SirT7 plays a key role, at least in the liver, in the control of autophagy in the physiological response to CR conditions. These results provide further evidence of a key role for the SirT7/mH2A axis in glucose/CR signaling. Overall, our findings not only suggest a key role for SirT7 in aging through gene expression regulation of second metabolism signaling but also suggest that this role is directly related to CR.

\section{DISCUSSION}

We report ADPRT activity in SirT7 and identify a novel ADPribosylation domain containing the ELHGN motif. The direct link between this modification, SirT7 dynamic distribution, and mH2A1 binding in response to GS provides a functional explanation of sirtuin enzymatic duality and suggests a key role for SirT7 in CR signaling and aging (Fig. 6C).

The catalytic duality of sirtuins has been widely debated since it was hypothesized that all sirtuins may have the ability to transfer ADP-ribosyl moieties to target proteins $(10,11)$. Originally described as a potential enzymatic alternative to the main deacylation/ deacetylation activity, it was assumed to act alongside the primary activity at the same catalytic site. This perception arose primarily because the ADPRT activity of some of the sirtuins tested was considerably weaker and less specific than the deacetylase activity and was dependent on the presence of an acetylated peptide $(11,34)$. Moreover, the dependency of this activity on the deacetylation reaction of yeast Sir2 was also reported, suggesting that it may be a side effect of primary catalytic deacylation or a technical artifact linked to the ability of sirtuins to hydrolyze $\mathrm{NAD}^{+}$as an intermediate step before deacetylation (35). It is of particular note that the vast majority of this evidence was based on in vitro ADP-ribosylation assays and was not validated by other techniques. In this sense, we report the first MS analysis of sirtuin ADPRTion and describe a consistent and reproducible pattern of N189-dependent ADPRTion in class IV sirtuins, the common lineage of SirT6 and SirT7 from early eukaryotes to mammals. We demonstrate not only that the role of the ELHGN motif in this activity is a highly conserved one but also that the catalytic site is physically separated, being located in a different structural domain. Our studies suggest that N189 could play an important role as the first acceptor of the ADP-ribose molecule generated by E185 before its transfer to the final target. This is supported by the direct interaction with E185 and the different behavior of N189Q compared with WT, despite its close structural similarity (fig. S1E). Given the wide distribution of these modified residues across the surface of SirT7, the automodification probably involves the intramodification of SirT7 oligomeric units within the SirT7 complex, similar to what has been described for PARP1 (36).

Although auto-ADPRTion had already been described in the early studies of sirtuin, its role has never been properly characterized. Our report suggests a direct role of this modification in SirT7 global distribution and in its chromatin-binding dynamics. PARP1 autoADPRTion was also proposed to regulate PARP1 chromatin-binding dynamics (37). As in the case of SirT7, mH2A1.1 has been also involved in PARP1 recruitment, although in this case, it was linked to PARP1 inactivation (38). Although our work has focused on autoADPRTion, the high degree of conservation within the class IV sirtuins and the considerable magnitude of the cavity, which makes it able to accommodate a wide range of proteins (Fig. 1E and fig. S1D), suggest that this novel domain may target other substrates.

In contrast to what was reported for SirT6-dependent ADPRTion of PARP1 and KAP1 $(16,17)$, SirT6 and SirT7 auto-mADPRTion does not depend on any key residues involved in the primary deacetylation domain, such as SirT6-S56/SirT7-S111, or SirT7-S115 (Fig. 1D and fig. S1B). This suggests that there may be two types of sirtuin-related ADP-ribosylation events, one that takes place at the primary catalytic site and that is probably directly associated with the main deacetylation reaction and another that is dependent on the ELHGN motif including auto-ADP-ribosylation and modification of other protein targets. This hypothesis suggests an interesting line of future work to confirm its validity and, thereafter, to define the extent and the specific range of substrates associated with each of these ADP-ribosylation events.

Another major finding of our work is the first evidence of a direct functional relationship between sirtuins and $\mathrm{mH} 2 \mathrm{~A}$ through this ADPR activity. This link is supported by the ability of $\mathrm{mH} 2 \mathrm{~A} 1.1$ to bind to the sirtuin product $O$-acetyl-ADP ribose (8). Although we have demonstrated that SirT7 auto-mADPRTion is essential for the interaction with $\mathrm{mH} 2 \mathrm{~A} 1.1$ between factors and the recruitment of SirT7 to these genomics sites, our observations suggest that there are other factors involved. Thus, while we detected auto-mADPRTion upon different types of stress such as GS, UV, and $\mathrm{H}_{2} \mathrm{O}_{2}$, we only 
A

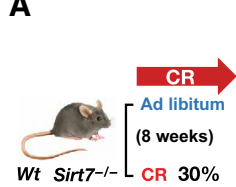

B

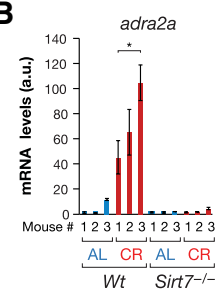

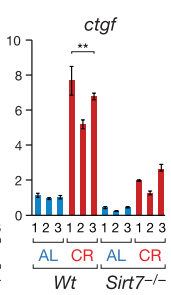
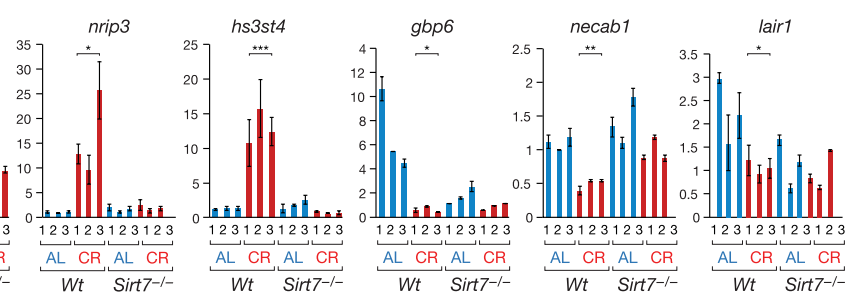

C

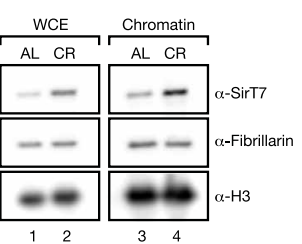

D

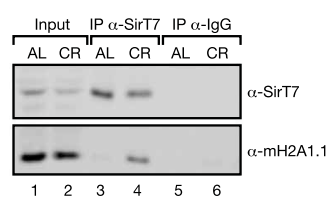

E
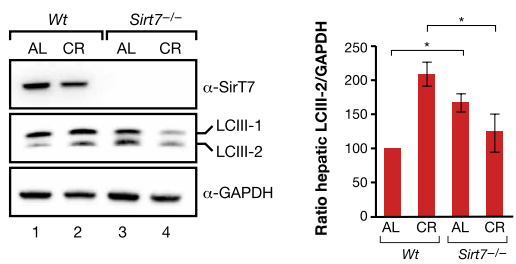

F

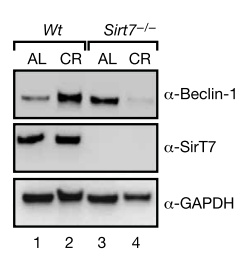

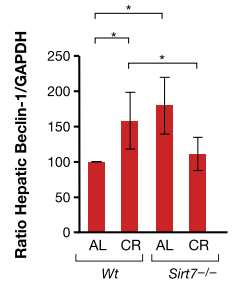

G

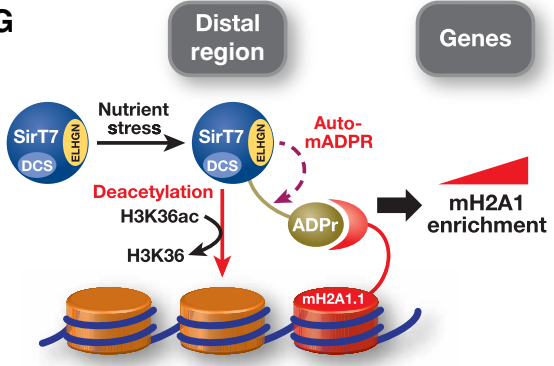

Genes

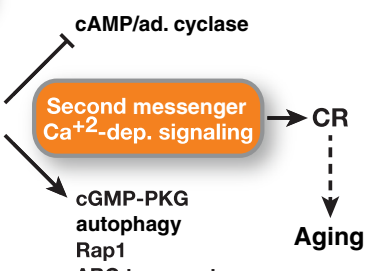

ABC transporters

Fig. 6. SirT7 and mH2A1 axis plays a key role in CR. (A) Model validation studies in WT and Sirt $7^{-/}$mice fed AL or calorie restricted (CR, 30\%) for 8 weeks. (B) RT-qPCR analysis of the indicated genes in liver samples from $W t$ and Sirt $7^{-1}$ mice fed AL or CR. Three animals were analyzed for each condition. Each quantification was generated from three replicates. Probabilities are those associated with one-way ANOVA ( ${ }^{*} P<0.05,{ }^{* *} P<0.01$, and $\left.{ }^{* *} P<0.005\right)$. (C) Levels of SirT7 in liver samples from $W t$ and Sirt $7^{-1-}$ mice AL and CR after subcellular fractionation. WCE and chromatin fractions are shown. (D) SirT7 immunoprecipitation of mH2A1 in the same liver samples. Inputs (I) and elutions (E) are shown. (E) Autophagy activity in the $W t$ and Sirt $7^{-1}$ livers under AL or CR monitored by levels of formation of LCIII-2. Left: A representative Western blot of $n=5$ replicates used in the quantification shown. Right: Quantification of the relative accumulation of LCIII-2 compared to glyceraldehyde-3-phosphate dehydrogenase (GAPDH) analyzed with a two-tailed $t$ test $\left({ }^{*} P<0.05\right)$. GAPDH was used as a loading control, as we did not detect a significant alteration of the levels of the protein in our conditions (data not shown). (F) Similar analysis $(n=5)$ of Beclin-1 as in (E). (G) Model proposed for the dual SirT7/mH2A regulatory axis in GS. On the basis of our data, we speculate that this axis is also involved in CR and aging. Nutrient stress induces SirT7 auto-mADPRT, which leads to mH2A-dependent recruitment of SirT7 to distal regulatory regions and subsequent $\mathrm{mH} 2 \mathrm{~A}$ enrichment around the associated genes. This axis plays a key role in $\mathrm{CR}$ in vivo and possibly in aging by modulating key signaling pathways.

observed a boost in the interaction between SirT7 and mH2A1 under GS conditions (Fig. 3F and fig. S3B). This suggested that there must be other signaling events involved that provide specific response under GS. Given the direct implication of stress-dependent kinases such as AMP kinase in the regulation of SirT7 exit from the nucleolus under energy deprivation conditions $(39,40)$, we speculate that phosphorylation of SirT7 may be also required to allow the observed activation of the SirT7/mH2A1 regulatory axis under these conditions. However, we cannot discard that other reported modifications of Sirt7, such as arginine methylation or acetylation, may be involved. Future studies should characterize these other signaling events involved in SirT7 activation upon GS and determine their contribution to the SirT7/mH2A1 functional relationship.

Our results demonstrate that GS triggers SirT7 relocalization mainly to distal regulatory regions mediated by mH2A1.1. These regions do not appear to be canonical enhancer regions because they are not enriched in enhancer-specific histone marks (data not shown). In turn, SirT7 promotes increased mH2A1 occupancy levels in the nearby gene, which results in the transcriptional activation or repression of the gene (Fig. 5). The fact that SirT7 seems to induce both up- and down-regulation in these genes suggests that its main role in this functional context is to promote $\mathrm{mH} 2 \mathrm{~A}$ enrichment, which, in turn, may regulate positive or negative gene expression.

The analysis of chromatin states suggests that $\mathrm{H} 3 \mathrm{~K} 27 \mathrm{me} 3$ is antagonistic to the interplay between SirT7 and mH2A. This intriguing observation deserves further study. The mechanism by which SirT7 promotes this long-range effect on $\mathrm{mH} 2 \mathrm{~A}$ enrichment around genes is unclear, and it may involve chromatin looping, as it occurs in enhancer regions. This possible mechanism is consistent with the recently established link between SirT7 and the nuclear lamina and chromatin organization (24). Our results suggest that the role of SirT7 in these genes requires both enzymatic activities, which further highlights the essential role of sirtuin duality in this regulatory mechanism. In agreement with this, we detected active deacetylation of H3K36 in the distal regulatory regions upon the arrival of SirT7, which may favor the mechanism of $\mathrm{mH} 2 \mathrm{~A}$ enrichment. However, our studies also suggest that in addition to this proposed mechanism, SirT7 may also regulate the expression of some of these target genes independently of the up-regulation of $\mathrm{mH} 2 \mathrm{~A} 1$. For instance, reexpression of H187Y or N189A in SirT7 ${ }^{-/-}$MEFs induced mild significant 
changes of expression in some of these genes upon GS without altering significantly the levels of $\mathrm{mH} 2 \mathrm{~A} 1$. Although, in the majority of these cases, this $\mathrm{mH} 2 \mathrm{~A} 1$-independent effect seems to be minor, this may reflect that both SirT7 enzymatic activities target other proteins in these genes. Since SirT7 has been shown to interact with a wide range of factors from chromatin enzymes to transcription regulators and target histone and nonhistone proteins, the observed effect may be due to a different combination of SirT7-associated factors depending on each specific gene context. Future studies should define these mechanisms, the factors involved, and their functional link to $\mathrm{mH} 2 \mathrm{~A} 1$.

This study also suggests that the Sirt $7 / \mathrm{mH} 2 \mathrm{~A}$ axis regulates a wide range of factors under GS. We were able to correlate the expression profile of these genes between GS stress conditions in primary MEFs and CR treatment in the livers of these mice. In these sense, we observed, as in GS, a SirT7-depenent expression of tested genes upon $\mathrm{CR}$, an increased localization of SirT7 in chromatin fractions, and a specific CR-dependent binding of SirT7 and mH2A1 in these livers (Fig. 6, C and D). Moreover, in agreement with previous studies, we detected not only gene expression profile differences associated with caloric restriction [e.g., ADRA2A, CTGF, and leukocyte-associated immunoglobulin-like receptor 1 (LAIR1)] (41) but also new potential markers involved in obesity [nuclear Receptor Interacting Protein 3 (NRIP3)] (42) and immune response [heparan sulfate-glucosamine 3-sulfotransferase 4 (HS3ST4) and GBP6] (43). Their stress-dependent expression response profile was SirT7 dependent, confirming the function of SirT7 as a nutrient sensor in response to calorie-restricted diet. The observed overrepresentation of second messenger signaling among these factors, particularly that related to calcium-associated signaling, is quite unexpected because it suggests that this is a major target of the SirT7/mH2A signaling axis. Considering the wide range of general and tissue-specific functions regulated by these pathways, the functional relevance of this regulatory axis may extend beyond glucose metabolism. cAMP/adenylate cyclase signaling was shown to regulate SirT6 activity, which suggests that regulatory feedback may operate in this context. SirT7 has been shown to regulate several key stress-dependent pathways such as p53, FOXO, Akt, mitogenactivated protein kinase, or extracellular signal-regulated kinase, among others $(27,44,45)$. The fact that the second messenger signaling plays an essential key role in many of these pathways suggests that SirT7 regulates these pathways through this SirT7/mH2A1 regulatory axis. Our studies suggest that the SirT7/mH2A1 signaling axis is involved in cellular adaptation to glucose deprivation. The studies with the CR model and the correlation that we have been able to establish between GS model in cell culture and the CR model in mouse lead us to speculate that the SirT7/mH2A1 interplay may have an important role in physiological short- and long-term adaptation to nutrient deprivation. This hypothesis should be further validated in future studies.

Another significant observation of our work is that SirT7 is important in the aging-associated changes linked to glucose homeostasis regulation. Although SirT7 was previously associated with aging at different levels, from genome stability and structure to ribosomal biogenesis and mitochondrial function $(20-22,24)$, our study is the first to establish a link between SirT7 and CR signaling. This link is supported by the in vivo metabolic alterations detected in SirT7 KO mice, such as reduced IGF-1 plasma levels and hepatic steatosis $(20,22)$. The decreased levels of autophagy observed in SirT7-deficient animals upon CR may also point directly to an effect on aging given the accelerated aging phenotype observed in $\mathrm{SirT}^{-1-}$ mice and that development of mammalian aging has been directly associated to a decreased autophagy activity. Under AL conditions, loss of SirT7 seems to promote an up-regulation of autophagy (Fig. 6, E and F), which may suggest that SirT7 plays an opposite role in autophagy in the absence of stress. Many genomic structural alterations caused by aging in mouse livers seem to take place in regions 50 to $500 \mathrm{~kb}$ upstream and downstream of the TSS of genes, which resembles the SirT7 binding profile upon GS. Some of the factors regulated by SirT7, such as CTGF or FoxA2, have been directly implicated in CR signaling and aging $(46,47)$. Further work is required to assess the contribution of these targets to the accelerated aging phenotype associated with SirT7. Our work not only offers a new insight into sirtuin duality and highlights the crucial role of sirtuins in the epigenetic regulation of metabolism and CR signaling but also provides a broader perspective on the dynamics and regulation of sirtuin function.

\section{MATERIALS AND METHODS}

\section{Animal studies, $\mathbf{C R}$, and studies}

For the CR procedure, 8-week-old male $\mathrm{Wt}$ and $\mathrm{SirT7}^{-/-}$animals (27) were housed in individual cages and randomly allocated into AL or CR group. The average of food eaten AL for each mouse was determined by weighing the remaining food on a daily basis for 1 week. The CR regimen was applied by feeding the animals with $70 \%$ of the calculated amount daily for 8 weeks. All animal experiments were approved by the institute's Institutional Animal Care and Use Committee and the local authorities (RP Darmstadt, Germany) and performed in accordance with the Guide for the Care and Use of Laboratory Animals published by the National Institutes of Health (NIH).

\section{SirT7 computational molecular model}

For in silico SirT7 structural model, an initial homology model was constructed for human SirT7 using the coordinates of the determined X-ray crystal structure of human SirT6 (Protein Data Bank ID: 5M6F; $1.87 \AA$ resolution, $42 \%$ of sequence identity). Modeller 9.12 was used to model the nondetermined regions of the protein. The side chain conformations for nonconserved residues were positioned according to SCWRL4 (Krivov). The protein was embedded in a TIP3P water box. The initial system was energy-minimized and subjected to $10 \mathrm{~ns}$ of molecular dynamics equilibration and, lastly, to a production stage extending to $150 \mathrm{~ns}$. All the simulations were performed with GROMACS 5.0 simulation package. The molecular model of SirT7 was used as the initial model to introduce H187Y and N189A mutations. For comparison purposes, SirT1 to SirT5 structures were superimposed to SirT6 structure and SirT7 structural model using PyMOL (The PyMOL Molecular Graphics System, version 1.8, Schrödinger LLC.).

\section{In vitro enzymatic assays}

ADP-ribosylation assays were performed in a total volume of $50 \mu \mathrm{l}$ in $50 \mathrm{mM}$ tris- $\mathrm{HCl}$ ( $\mathrm{pH} 7.8$ ), $1 \mathrm{mM} \mathrm{MgCl}, 0.3 \mathrm{mM}$ dithiothreitol (DTT), $1 \mu \mathrm{M}$ unlabeled $\mathrm{NAD}^{+}$, and $8 \mu \mathrm{Ci}$ of $\left[{ }^{32} \mathrm{P}\right]-\mathrm{NAD}^{+}(800 \mathrm{Ci} / \mathrm{mmol}$; PerkinElmer) incubated with 3 to $5 \mu \mathrm{g}$ of recombinant protein from Escherichia coli (BL21) or purified from HEK293F cells. The reactions were incubated at least for 3 hours at $37^{\circ} \mathrm{C}$. Reactions were stopped by adding $5 \times$ Laemmli sample buffer (supplemented with $10 \%$ 2-mercaptoethanol) to a final concentration of $1 \times$ and then separated by SDS-polyacrylamide gel electrophoresis. Following gel electrophoresis, 
proteins were then transferred from gel to PVDF membrane and then stained with Coomassie Brilliant Blue (Sigma-Aldrich). The incorporation of ADP-ribosylation into proteins was visualized by autoradiography.

Deacetylation assays for SirT6 were performed in $50 \mathrm{mM}$ tris$\mathrm{HCl}\left(\mathrm{pH} \mathrm{8.0)}, 150 \mathrm{mM} \mathrm{NaCl}\right.$, and $1 \mathrm{mM} \mathrm{MgCl}_{2}$ with $2 \mathrm{mM} \mathrm{NAD}^{+}$ (Sigma-Aldrich) in the presence of $3 \mu \mathrm{g}$ of rSirT6 and $1 \mu \mathrm{g}$ of semisynthetic recombinant mononucleosomes containing acetyl-lysine at position 18 of histone $\mathrm{H} 3$ (H3K18ac dNuc, EpiCypher) for 5 hours at $30^{\circ} \mathrm{C}$. In the case of SirT7, reactions were performed in $50 \mathrm{mM}$ tris- $\mathrm{HCl}$ (pH 7.8), $1 \mathrm{mM} \mathrm{MgCl}_{2}$, and $0.3 \mathrm{mM}$ DTT with $5 \mathrm{mM} \mathrm{NAD}^{+}$ with 3 to $5 \mu \mathrm{g}$ of SirT7 and $\sim 0.5 \mu \mathrm{g}$ of hyperacetylated histone substrates for 3 hours at $37^{\circ} \mathrm{C}$. The acetylation status was determined by Western blot analysis using specific antibodies.

\section{Recombinant protein purification}

Human cDNAs of SirT6 or SirT7 were cloned into the pET-30b expression vector (Novagen, Germany), containing a hexahistidine tag at the $\mathrm{C}$ terminus. The constructs were expressed in E. coli BL21 (DE3) grown at $37^{\circ} \mathrm{C}$ unit optical density at $600 \mathrm{~nm}$ of 0.5 and then induced with $1 \mathrm{mM}$ isopropyl- $\beta$-D-thiogalactopyranoside for 4 hours. Harvested cells were resuspended in lysis buffer $[20 \mathrm{mM}$ Hepes ( $\mathrm{pH} 7.9)$, $500 \mathrm{mM} \mathrm{NaCl}, 10 \%$ glycerol, 1 mM EDTA (pH 8.0), 0.1\% NP-40, 20 $\mathrm{mM}$ 2-mercaptoetanol, and $1 \mathrm{mM}$ phenylmethylsulfonyl fluoride (PMSF) ] and lysed by sonication. After centrifugation at $12,000 \mathrm{~g}$ at $4^{\circ} \mathrm{C}$ for 1 hour, the cleared lysates were loaded onto $\mathrm{Ni}$-nitrilotriacetic acid agarose resin (Qiagen) by gravity flow. After several washes with lysis buffer and BC500 [20 mM tris- $\mathrm{HCl}(\mathrm{pH} 8.0), 500 \mathrm{mM}$ $\mathrm{KCl}, 10 \%$ glycerol, $1 \mathrm{mM}$ EDTA, $1 \mathrm{mM}$ DTT, $0.1 \%$ Nonidet P-40, and $0.2 \mathrm{mM}$ PMSF], rSirT7 was eluted using $100 \mathrm{mM}$ imidazole and subsequently dialyzed in $\mathrm{BC} 100$. Recombinant proteins were then kept at $-80^{\circ} \mathrm{C}$.

Preparation of recombinant histones were performed from E. coli Bl21 (DE3) cells as described elsewhere (48). Histone octamers were concentrated to 3 to $15 \mathrm{mg} / \mathrm{ml}$, adjusted to $50 \%$ (v/v) glycerol, and stored at $-80^{\circ} \mathrm{C}$.

\section{Cells transfections and treatments}

MEFs derived from $W t$ and SirT7 KO mice were generated from day 13.5 embryos by standard methods. For retroviral infection of primary MEFs and NIH3T3 cells, Platinum-A cells were transiently transfected using polyethylenimine. Cells were incubated with virus-containing supernatant in the presence of polybrene $(8 \mu \mathrm{g} / \mathrm{ml})$. In the case of shRNA in NIH3T3 cells, after 48 hours of infection, infected cells were selected for 72 hours with puromycin $(2.0 \mu \mathrm{g} / \mathrm{ml})$. Plasmids encoding mouse shRNAs of $\mathrm{mH} 2 \mathrm{~A}$ isoforms were obtained from Addgene (Cambridge, MA, USA): pSUPER retro puro mH2A1 shRNA (\#30517) and pSUPER retro puro Scr shRNA (\#30520). HEK293F cells were transiently transfected with plasmids using $3 \mu \mathrm{l}$ of polyethylenimine $(1 \mathrm{mg} / \mathrm{ml})$ per $\mu \mathrm{g}$ of DNA. The following plasmids were used for transfection: pcDEF-Flag-SirT7 (WT, H187Y, N189A, and N189Q) and pCMV6-SirT7-green fluorescent protein (GFP) (OriGene, RG205658).

For GS treatments, MEFs, NIH3T3, and HEK293F cells were treated 24 to 40,12 , and 4 hours, respectively, before harvesting with glucose-free Dulbecco's modified Eagle's medium (Gibco) containing $10 \%$ dialyzed fetal bovine serum. For oxidative stress, cells were treated with $1 \mathrm{mM} \mathrm{H}_{2} \mathrm{O}_{2}$ for 1 hour before harvesting. Cells were irradiated by exposure in the following conditions: (i) UV irradiation at $254 \mathrm{~nm}\left(100 \mathrm{~J} / \mathrm{m}^{2}\right)$ in a Stratagene Stratalinker $2400 \mathrm{UV}$ (Agilent Technologies) and (ii) gamma irradiation delivered using an aluminum filter at $100 \mathrm{kV}$ peak in a Faxitron cabinet $\mathrm{x}$-ray system (Faxitron Corp.).

\section{Immunoprecipitations and Western blot}

Whole-cell proteins and nuclear extracts were prepared according to the Dignam protocol. Nuclear extracts were treated with Benzonase (Sigma-Aldrich), cOmplete Protease Inhibitor (Roche), and $2 \mathrm{mM}$ $\mathrm{MgCl}_{2}$ for 6 hours at $4^{\circ} \mathrm{C}$. The lysate was clarified by centrifugation, and protein supernatant was incubated with the respective crosslinking primary antibody to Protein G Agarose resin (EMD Millipore). The cross-linking was performed in $20 \mathrm{mM}$ dimethyl pimelimidate (DMP) (Pierce, Thermo Fisher Scientific, MA, USA) dissolved in $0.2 \mathrm{M}$ sodium borate ( $\mathrm{pH} 9.0$ ). After quenching with $0.2 \mathrm{M}$ ethanolamine ( $\mathrm{pH} 8.0)$, the cross-linked beads with the antibody were stored at $4^{\circ} \mathrm{C}$ in phosphate-buffered saline (PBS) until use. The affinitypurified protein complexes from HEK293F cells were gently washed at least three time with buffer BC300 [20 mM tris- $\mathrm{HCl}(\mathrm{pH} 8.0)$ $300 \mathrm{mM} \mathrm{KCl}, 10 \%$ glycerol, $1 \mathrm{mM}$ EDTA, 1 mM DTT, $0.05 \%$ Nonidet $\mathrm{P}-40$, and $0.1 \mathrm{mM}$ PMSF]. Purification of tagged proteins was done at least five times with BC500 [20 mM tris- $\mathrm{HCl}(\mathrm{pH} 8.0) 500 \mathrm{mM}$ $\mathrm{KCl}, 10 \%$ glycerol, $1 \mathrm{mM}$ EDTA, $1 \mathrm{mM}$ DTT, $0.1 \%$ Nonidet P-40, and $0.1 \mathrm{mM}$ PMSF] and then eluted either with synthetic peptide $(0.4 \mu \mathrm{g} / \mathrm{ml})$ from GenScript (Piscataway, NJ, USA) or by acidification using a buffer containing $0.2 \mathrm{M}$ glycine ( $\mathrm{pH} 2.3$ ).

Endogenous SirT7 immunoprecipitated from liver tissues were performed by using a Polytron homogenizer in ice-cold radioimmunoprecipitation assay buffer [50 mM tris- $\mathrm{HCl}$ ( $\mathrm{pH} 8.0), 150 \mathrm{mM}$ $\mathrm{NaCl}, 1 \% \mathrm{NP}-40,0.25 \%$ sodium deoxycholate, and cOmplete Protease Inhibitor (Roche)]. Whole-cell extract was treated with Benzonase for 6 hours in $1 \mathrm{mM} \mathrm{MgCl} 2$ at $4^{\circ} \mathrm{C}$ and then sonicated on ice. After centrifugation at $17,000 \mathrm{~g}$ for $5 \mathrm{~min}$ at $4^{\circ} \mathrm{C}$, protein extracts were incubated with anti-SirT7 cross-linked beads by gently rotating at $4^{\circ} \mathrm{C}$ overnight. After three washes with ice-cold BC350 and $0.075 \%$ NP40, SirT7 and Sirt7-interacting proteins were eluted from the resin with $0.2 \mathrm{M}$ glycine ( $\mathrm{pH}$ 2.3). Coimmunoprecipitation experiments between rSirT7 WT and N189A (preincubated $\pm \mathrm{NAD}^{+}$) with nuclear extracts from HEK293F SirT7 KO cells (CRISPR-Cas9-mediated $\mathrm{KO}$ of SIRT7) expressing hemagglutinin (HA)-mH2A1.1 WT or G224E were performed in BC300. After three washes with ice-cold BC350 and $0.075 \%$ NP-40, proteins were eluted from the resin with $0.2 \mathrm{M}$ glycine ( $\mathrm{pH} 2.3$ ). Densitometric analysis of the Western blots was performed with ImageJ software.

The following antibodies were used for Western blotting: anti-Flag M2 Antibody (F1804, Sigma-Aldrich), anti-fibrillarin (B-1, Sc-166001, Santa Cruz Biotechnology), anti-H3 (\#9715, Cell Signaling Technology), anti-tubulin (ab-6160, Abcam), anti-glyceraldehyde-3-phosphate dehydrogenase (\#2118, Cell Signaling Technology), anti-SirT7 (\#5360, Cell Signaling Technology), anti-SirT7 (sc-365344, Santa Cruz Biotechnology), anti-mH2A1.1 (D5F6N, Cell Signaling Technology), anti-mH2A1.2 (\#4827, Cell Signaling Technology), anti-mH2A1 (ab37264, Abcam), anti-mH2A.2 (gift from M.B.), anti-histone H2A (\#12349, Cell Signaling Technology), anti-HA (H6908, SigmaAldrich), anti-myc (\#2276, Cell Signaling Technology), anti-H3K18ac (\#9675, Cell Signaling Technology), anti-H3K27me3 (\#9733, Cell Signaling Technology), anti-LC3B (L7543, Sigma-Aldrich); antiBeclin-1 (\#3738, Cell Signaling Technology), and anti-pan-ADPribose binding reagent (MABE1016, Millipore). 


\section{Subcellular fractionation}

To determine the amount of SirT7 proteins in the cytoplasm, nucleoplasm, and tight chromatin fraction, NIH3T3 cells were transfected with SirT7-HA WT, H187Y, N189Q, and N189A. Cytoplasmic and nucleoplasm fraction were obtained with the Dignam protocol extraction (fractions $\mathrm{A}$ and $\mathrm{C}$ ), and the remaining chromatin fraction was then solubilized in $1 \times$ Laemmli buffer and sonicated with Branson 250 sonicator.

Preparation of subcellular fractions from the mouse liver tissue was prepared by using a Polytron homogenizer in buffer $\mathrm{H}[20 \mathrm{mM}$ tris- $\mathrm{HCl}$ (pH 7.4), $0.25 \mathrm{mM}$ sucrose, $3 \mathrm{mM} \mathrm{CaCl}$, and cOmplete Protease Inhibitor]. The nuclei were washed three times by centrifugation at $300 \mathrm{~g}$ at $4^{\circ} \mathrm{C}$ for $5 \mathrm{~min}$. The nuclear pellet was resuspended by vortexing for $5 \mathrm{~s}$ in buffer $\mathrm{C}$ and incubated for $10 \mathrm{~min}$ on ice and then centrifuged at $17,000 \mathrm{~g}$ at $4^{\circ} \mathrm{C}$ for $10 \mathrm{~min}$. The resultant pellet considered as the chromatin fraction was solubilized in $1 \times$ Laemmli buffer and then sonicated, whereas the supernatant was considered as the nucleoplasmic fraction.

\section{Mass spectrometric analysis}

Protein extracts were precipitated with cold acetone $\left(\mathrm{o} / \mathrm{n},-20^{\circ} \mathrm{C}\right)$, and the protein pellets were resuspended in $20 \mu \mathrm{l}$ of $200 \mathrm{mM}$ ammonium bicarbonate with $6 \mathrm{M}$ urea solution. Samples were reduced with DTT $\left(10 \mathrm{mM}, 30^{\circ} \mathrm{C}, 60 \mathrm{~min}\right)$ and alkylated with chloroacetamide $\left(20 \mathrm{mM}, 25^{\circ} \mathrm{C}, 30 \mathrm{~min}\right)$. The samples were diluted with $\mathrm{NH}_{4} \mathrm{HCO}_{3}$ to reduce urea concentration below $1 \mathrm{M}$ and digested with trypsin ( $1: 10$ enzyme:protein, $30^{\circ} \mathrm{C}, 16$ hours; Promega). After digestion, the peptide mix was acidified with formic acid and then desalted with a MicroSpin C18 column (The Nest Group) before liquid chromatography-tandem MS (LC-MS/MS) analysis.

The peptide samples were analyzed using a Lumos Orbitrap mass spectrometer (Thermo Fisher Scientific, San Jose, CA, USA) coupled to an EASY-nLC 1000 [Thermo Fisher Scientific (Proxeon), Odense, Denmark]. Afterward, peptides were loaded directly onto the analytical column and were separated by reversed-phase chromatography using a $50-\mathrm{cm}$ column with an inner diameter of $75 \mu \mathrm{m}$, packed with a $2-\mu \mathrm{m} \mathrm{C18}$ particle spectrometer (Thermo Fisher Scientific, San Jose, CA, USA) and a binary solvent system of $0.1 \%$ formic acid in $\mathrm{H}_{2} \mathrm{O}$ (solvent $\mathrm{A}$ ) and $0.1 \%$ formic acid in acetonitrile (solvent B). Chromatographic gradients started at $7 \% \mathrm{~B}$ with a flow rate of $300 \mathrm{nl} / \mathrm{min}$ and gradually increased to $22 \% \mathrm{~B}$ in $52 \mathrm{~min}$, then to $32 \% \mathrm{~B}$ in $8 \mathrm{~min}$, and to $95 \% \mathrm{~B}$ in $10 \mathrm{~min}$. After each analysis, the column was washed for 10 min with $95 \% \mathrm{~B}$.

The acquisition was performed in data-dependent acquisition mode, and full MS scans with 1 microscan at a resolution of 120,000 were used over a mass range of $m / z 300$ to 1500 . Precursor automated gain control (AGC) was set to $2 \times 10^{5}$. Charge-state screening was enabled, and precursors with +2 to +5 charge states and intensities $>5 \times 10^{4}$ were selected for MS/MS and fragmented as previously described (49).Briefly, precursors that exhibited more than two ADP-ribose fragment peak $(136,0623,250,0940,348,0709$, and 428,0372) after an initial high-energy collisional dissociation (HCD) fragmentation were submitted to a high-quality HCD fragmentation event (acquired in the Orbitrap with collision energy of 35\% and a resolution of 120,000; AGC was set to $5 \times 10^{5}$ isolation window of $2 \mathrm{~m} / \mathrm{z}$ and maximum injection time of $240 \mathrm{~ms}$ was used) and an electrontransfer/higher-energy collision dissociation MS/MS (the "use-calibrated charge-dependent parameter" option was selected, and it was acquired in the Orbitrap with a supplementary activation (SA) of $25 \%$ and a resolution of 120,000 ; AGC was set to $5 \times 10^{5}$; isolation window of $2 \mathrm{~m} / \mathrm{z}$ and maximum injection time of $240 \mathrm{~ms}$ were used). Precursors masses selected in previous cycle fragmentation were excluded for further selection for $60 \mathrm{~s}$, and the exclusion window was set at 10 parts per million (ppm). Analysis of SirT6WT/S56A in vitro assays and of SirT7 under stress conditions were performed using an Eclipse Orbitrap mass spectrometer (Thermo Fisher Scientific, San Jose, CA, USA) coupled to an EASY-nLC 1200 [Thermo Fisher Scientific (Proxeon), Odense, Denmark]. See more details in the Supplementary Materials related to the chromatography parameters and MS/MS acquisition method (Supplementary Materials and Methods).

Acquired spectra were analyzed using the PEAKS X+ software, which, as a default, performs de novo peptide sequencing before database searches, to improve the accuracy of the results. The data were searched against a Swiss-Prot human database (as of October 2019). Trypsin (specific, up to three missed cleavages allowed) was selected for database searches, and no enzyme was chosen in de novo searches (up to five candidates per spectrum reported). The maximal mass error was set to $10 \mathrm{ppm}$ for precursor ions and $0.05 \mathrm{Da}$ for product ions. Carbamidomethylation was selected as a fixed modification, and methionine oxidation and ADP-ribose $(+541.0611 \mathrm{Da}$ at $\mathrm{C}, \mathrm{D}$, $\mathrm{E}, \mathrm{H}, \mathrm{K}, \mathrm{N}, \mathrm{Q}, \mathrm{R}, \mathrm{S}, \mathrm{T}$, and Y) (50) were set as variable modifications. The maximal number of modifications per peptide was set to three. The false discovery rate was set to 0.05 for peptides.

\section{RNA isolation, cDNA synthesis, and real-time quantitative polymerase chain reactions}

Total RNA was purified from MEFs and liver using TRIzol reagent (Invitrogen, Carlsbad, CA, USA). The cDNA was synthesized from $5 \mu \mathrm{g}$ of total RNA with a Transcriptor First Strand cDNA Synthesis Kit (Roche) according to the manufacturer's instructions. Real-time quantitative polymerase chain reaction (RT-qPCR) was performed using the QuantStudio 5 Real-Time PCR System (Thermo Fisher Scientific) with SYBR Green PCR Master Mix of Applied Biosystems. Relative gene expression was analyzed in QuantStudio 5 software (Thermo Fisher Scientific), and values were normalized to the expression of RNF219 in the case of CR and GS treatments. Details of oligonucleotides are shown in table S4.

\section{Site-directed mutagenesis}

The different constructs harboring point mutations were cloned by site-directed mutagenesis on the basis of the QuikChange protocol (Stratagene). The positive clones selected were then verified by sequencing using a 3730 DNA Analyzer (Applied Biosystems, Foster City, CA, USA).

\section{Immunofluorescence}

$\mathrm{SirT7}^{-/-}$cells were fixed with $4 \%$ paraformaldehyde for $7 \mathrm{~min}$ at room temperature and permeabilized for 5 min with buffer A [ $1 \%$ bovine serum albumin (BSA) and $0.5 \%$ Triton in PBS]. Cells were blocked with $1 \%$ BSA-PBS solution for 1 hour and further incubated with a rabbit anti-B23 antibody (1:100; Sc-6013-R, Santa Cruz Biotechnology) for 1 hour in buffer B (1\% BSA and $0.2 \%$ Triton in PBS). Cells were washed three times 15 min each with PBS and stained with an Alexa Fluor 555 fluorophore-conjugated secondary antibody (1:200; A27039, Life Technologies). Fluorescence images were acquired with a Zeiss 710 confocal module coupled to an Axio Observer Z1 equipped with a $63 \times$ oil immersion objective. Image analysis was performed using ImageJ $(\mathrm{NIH})$. 


\section{ChIP-seq and RNA-seq}

For RNA-seq, total RNA from $10-\mathrm{cm}$ dishes containing $W t$ and Sir $\mathrm{T7}^{-/-}$exponentially growing MEFs was harvested using an RNeasy miniprep kit (Qiagen) according to the manufacturer's instructions. Samples were sent to the RUCDR, Infinite Biologics sequencing center (Rutgers University, NJ, USA) for RNA extraction, library preparation (Illumina TruSeq chemistry), and sequencing [ $2 \times 100$-base pair (bp) paired-end reads, $>60 \times 106$ fragments per sample, Illumina HiSeq in rapid mode]. Reads were demultiplexed, concatenated, and delivered as FASTQ files. Raw reads were assessed for quality with FastQC. Raw reads were trimmed with Trimmomatic, aligned to the University of California Santa Cruz (UCSC) $\mathrm{mm} 9$ reference genome with HISAT2 (options: --dta), and converted to sorted and indexed BAM format with SAMtools. StringTie (in -eB mode) was used to estimate transcript abundance using the UCSC $\mathrm{mm} 9$ reference GTF file (obtained from the Illumina iGenomes UCSC mm9 bundle). Differential expression was analyzed with DESeq2, allowing modeling terms for genotype, treatment, and batch.

ChIP was conducted as described before (20). Briefly, nuclear extracts from $W t$ and $\mathrm{SirT}^{-/-}$cells were first obtained, and chromatin was prepared by sonication to an average fragment size of $300 \mathrm{bp}$. For SirT7 ChIP, chromatin was diluted eight times in dilution buffer, and for macro-H2A and $\mathrm{H} 3 \mathrm{~K} 27 \mathrm{~K} 3 \mathrm{~m} 3 \mathrm{ChIP}$, chromatin was diluted five times. Antibodies were conjugated to beads, and ChIP was conducted overnight at $4^{\circ} \mathrm{C}$ on a rotator. After washes and reversal of cross-links, ChIP libraries were prepared using a ThruPLEX DNAseq library preparation kit (Rubicon Genomics) following the manufacturer's protocol. ChIP libraries were then size-selected with Pippin Prep (Sage Science) for a final library fragment size of 350 to $400 \mathrm{bp}$ and quantified with KAPA qPCR. ChIP libraries were then sequenced on an Illumina HiSeq 2500 with $2 \times 100$ or $2 \times 75$ bp paired-end reads to a depth of $>20 \times 10^{6}$ fragments per sample at the RUCDR, Infinite Biologics sequencing center (Rutgers University, NJ, USA). Reads were demultiplexed, concatenated, and delivered as FASTQ files.

Raw read quality was first assessed with FastQC version 0.11.3, followed by adapter clipping and quality trimming with Trimmomatic version 0.36. Reads were then aligned to the mouse (UCSC mm9) reference genome with Bowtie with options $-\mathrm{n} 2-\mathrm{k} 10$ and converted to BAM format with SAMtools. ChIP, library, and alignment quality was evaluated by PCR bottleneck coefficient (PBC), fraction of reads in peaks (FRiP), insert size, and strand cross-correlation analysis. All samples passed the ENCODE quality standards for ChIP-seq studies. Reads per kilobase per million mapped reads-normalized signal files were generated from BAM data using deepTools version 2.4.2 using a bin size of $10 \mathrm{bp}$.

SirT7 peaks were called in each biological replicate independently using MACS version 1.4.2 (--keep-dup auto --bw 450 --pvalue 0.05). Reproducibility of individual peaks was assessed with irreproducible discovery rate (IDR) (version 2.0.2), and the peaks passing a $-\log 10$ (global IDR) threshold of 1.3 across biological replicates were kept. SirT7-associated genes were called using the GREAT tool with IDR-passing peaks and the association rule of "Basal plus extension" with parameters Proximal $=5.0 \mathrm{~kb}$ upstream, $1.0 \mathrm{~kb}$ downstream, plus Distal up to $10.0 \mathrm{~kb}$, including curated regulatory domains. For mH2A1.1 and H3K27me3 ChIP-seq datasets, we used ChromHMM to segment the genome into four learned states. First, BAM files for ChIP and corresponding INPUT samples were binarized at a resolution of 200-bp bins. Binarized data were submitted to the ChromHMM
LearnModel program and allowed to learn four states. The learned model showed posterior enrichment for the following states: U1, no enrichment; U2, mH2A1 enrichment; U3, H3K27me3 enrichment; and $\mathrm{U} 4$, both $\mathrm{mH} 2 \mathrm{~A} 1$ and $\mathrm{H} 3 \mathrm{~K} 27 \mathrm{me} 3$ enrichment. mH2A1-enriched regions were considered as the union of regions identified as states $\mathrm{U} 2$ and $\mathrm{U} 4$. mH2A1-enriched genes were called using the GREAT tool with $\mathrm{mH} 2 \mathrm{~A} 1$ regions as input with the association rule of "single nearest gene" within $10 \mathrm{~kb}$, including curated regulatory domains. Chromatin state transitions induced by GS treatment were computed relative to NT for each genotype. Briefly, we identified 200-bp bins in GS with differing state calls than NT and recorded the initial state (that occurring in the NT) and the final state (that occurring in the GS). We then took the subset of transition sites at the intersection of gene lists ( $\pm 3 \mathrm{~kb}$ upstream and downstream of each gene). We quantified transition occupancy by counting the number of base pairs occupied by each transition type and normalized this by the total number of base pairs that underwent any transition. To ascertain the genomic context of these transitions, we submitted each transition type to the CEAS tool.

\section{Statistical analysis}

The represented values show means of at least three or more independent experiments ( $n \geq 3$ with error bars representing SEM unless otherwise specified). Data were analyzed using a two-tailed Student's $t$ test (ADP-ribosylation and deacetylation assays and RT-qPCRs from rescue experiments in MEFs), and for multiple comparisons (RT-qPCRs in mice under caloric restriction), the $P$ value was calculated using Microsoft Excel software by two-way analysis of variance (ANOVA).

\section{SUPPLEMENTARY MATERIALS}

Supplementary material for this article is available at http://advances.sciencemag.org/cgi/ content/full/6/30/eaaz2590/DC1

View/request a protocol for this paper from Bio-protocol.

\section{REFERENCES AND NOTES}

1. L. Bosch-Presegué, A. Vaquero, Sirtuins in stress response: Guardians of the genome. Oncogene 33, 3764-3775 (2014).

2. P. Martínez-Redondo, A. Vaquero, The diversity of histone versus nonhistone sirtuin substrates. Genes Cancer 4, 148-163 (2013).

3. H. Jiang, S. Khan, Y. Wang, G. Charron, B. He, C. Sebastián, J. Du, R. Kim, E. Ge, R. Mostoslavsky, H. C. Hang, Q. Hao, H. Lin, SIRT6 regulates TNF- $\alpha$ secretion through hydrolysis of long-chain fatty acyl lysine. Nature 496, 110-113 (2013).

4. C. E. Caban, A. Ginsburg, Glutamine synthetase adenylyltransferase from Escherichia coli: Purification and physical and chemical properties. Biochemistry 15, 1569-1580 (1976).

5. O. Nureki, D. G. Vassylyev, M. Tateno, A. Shimada, T. Nakama, S. Fukai, M. Konno, T. L. Hendrickson, P. Schimmel, S. Yokoyama, Enzyme structure with two catalytic sites for double-sieve selection of substrate. Science 280, 578-582 (1998).

6. M. O. Hottiger, Nuclear ADP-ribosylation and its role in chromatin plasticity, cell differentiation, and epigenetics. Annu. Rev. Biochem. 84, 227-263 (2015).

7. G. Kustatscher, M. Hothorn, C. Pugieux, K. Scheffzek, A. G. Ladurner, Splicing regulates NAD metabolite binding to histone macroH2A. Nat. Struct. Mol. Biol. 12, 624-625 (2005)

8. V. Pazienza, M. Borghesan, T. Mazza, F. Sheedfar, C. Panebianco, R. Williams, G. Mazzoccoli, A. Andriulli, T. Nakanishi, M. Vinciguerra, SIRT1-metabolite binding histone macroH2A1.1 protects hepatocytes against lipid accumulation. Aging 6, 35-47 (2014).

9. M. J. Gamble, W. L. Kraus, Multiple facets of the unique histone variant macroH2A: From genomics to cell biology. Cell Cycle 9, 2568-2574 (2010).

10. R. A. Frye, Characterization of five human CDNAs with homology to the yeast SIR2 gene: Sir2-like proteins (sirtuins) metabolize NAD and may have protein ADP-ribosyltransferase activity. Biochem. Biophys. Res. Commun. 260, 273-279 (1999).

11. J. C. Tanny, G. J. Dowd, J. Huang, H. Hilz, D. Moazed, An enzymatic activity in the yeast Sir2 protein that is essential for gene silencing. Cell 99, 735-745 (1999).

12. K. Fahie, P. Hu, S. Swatkoski, R. J. Cotter, Y. Zhang, C. Wolberger, Side chain specificity of ADP-ribosylation by a sirtuin. FEBS J. 276, 7159-7176 (2009). 
13. B. J. North, B. L. Marshall, M. T. Borra, J. M. Denu, E. Verdin, The human Sir2 ortholog, SIRT2, is an NAD ${ }^{+}$-dependent tubulin deacetylase. Mol. Cell 11, 437-444 (2003).

14. P. W. Pan, J. L. Feldman, M. K. Devries, A. Dong, A. M. Edwards, J. M. Denu, Structure and biochemical functions of SIRT6. J. Biol. Chem. 286, 14575-14587 (2011).

15. M. C. Haigis, R. Mostoslavsky, K. M. Haigis, K. Fahie, D. C. Christodoulou, A. J. Murphy, D. M. Valenzuela, G. D. Yancopoulos, M. Karow, G. Blander, C. Wolberger, T. A. Prolla, R. Weindruch, F. W. Alt, L. Guarente, SIRT4 inhibits glutamate dehydrogenase and opposes the effects of calorie restriction in pancreatic $\beta$ cells. Cell 126, 941-954 (2006).

16. Z. Mao, C. Hine, X. Tian, M. Van Meter, M. Au, A. Vaidya, A. Seluanov, V. Gorbunova, SIRT6 promotes DNA repair under stress by activating PARP1. Science 332, 1443-1446 (2011).

17. M. Van Meter, M. Kashyap, S. Rezazadeh, A. J. Geneva, T. D. Morello, A. Seluanov, V. Gorbunova, SIRT6 represses LINE1 retrotransposons by ribosylating KAP1 but this repression fails with stress and age. Nat. Commun. 5, 5011 (2014).

18. G. Liszt, E. Ford, M. Kurtev, L. Guarente, Mouse Sir2 homolog SIRT6 is a nuclear ADP-ribosyltransferase. J. Biol. Chem. 280, 21313-21320 (2005).

19. M. F. Barber, E. Michishita-Kioi, Y. Xi, L. Tasselli, M. Kioi, Z. Moqtaderi, R. I. Tennen, S. Paredes, N. L. Young, K. Chen, K. Struhl, B. A. Garcia, O. Gozani, W. Li, K. F. Chua, SIRT7 links H3K18 deacetylation to maintenance of oncogenic transformation. Nature 487, 114-118 (2012).

20. B. N. Vazquez, J. K. Thackray, N. G. Simonet, N. Kane-Goldsmith, P. Martínez-Redondo, T. Nguyen, S. Bunting, A. Vaquero, J. A. Tischfield, L. Serrano, SIRT7 promotes genome integrity and modulates non-homologous end joining DNA repair. EMBO J. 35 , 1488-1503 (2016).

21. E. Ford, R. Voit, G. Liszt, C. Magin, I. Grummt, L. Guarente, Mammalian Sir2 homolog SIRT7 is an activator of RNA polymerase I transcription. Genes Dev. 20, 1075-1080 (2006).

22. D. Ryu, Y. S. Jo, G. Lo Sasso, S. Stein, H. Zhang, A. Perino, J. U. Lee, M. Zeviani, R. Romand, M. O. Hottiger, K. Schoonjans, J. Auwerx, A SIRT7-dependent acetylation switch of GABP $\beta 1$ controls mitochondrial function. Cell Metab. 20, 856-869 (2014).

23. W. W. Wang, M. Angulo-lbanez, J. Lyu, Y. Kurra, Z. Tong, B. Wu, L. Zhang, V. Sharma, J. Zhou, H. Lin, Y. Q. Gao, W. Li, K. F. Chua, W. R. Liu, A click chemistry approach reveals the chromatin-dependent histone H3K36 deacylase nature of SIRT7. J. Am. Chem. Soc. 141, 2462-2473 (2019).

24. B. N. Vazquez, J. K. Thackray, N. G. Simonet, S. Chahar, N. Kane-Goldsmith, S. J. Newkirk, S. Lee, J. Xing, M. P. Verzi, W. An, A. Vaquero, J. A. Tischfield, L. Serrano, SIRT7 mediates L1 elements transcriptional repression and their association with the nuclear lamina. Nucleic Acids Res. 47, 7870-7885 (2019).

25. T. Yoshizawa, M. F. Karim, Y. Sato, T. Senokuchi, K. Miyata, T. Fukuda, C. Go, M. Tasaki, K. Uchimura, T. Kadomatsu, Z. Tian, C. Smolka, T. Sawa, M. Takeya, K. Tomizawa, Y. Ando, E. Araki, T. Akaike, T. Braun, Y. Oike, E. Bober, K. Yamagata, SIRT7 controls hepatic lipid metabolism by regulating the ubiquitin-proteasome pathway. Cell Metab. 19, 712-721 (2014).

26. J. Fang, A. lanni, C. Smolka, O. Vakhrusheva, H. Nolte, M. Krüger, A. Wietelmann, N. G. Simonet, J. M. Adrian-Segarra, A. Vaquero, T. Braun, E. Bober, Sirt7 promotes adipogenesis in the mouse by inhibiting autocatalytic activation of Sirt1. Proc. Natl. Acad. Sci. U.S.A. 114, E8352-E8361 (2017).

27. O. Vakhrusheva, C. Smolka, P. Gajawada, S. Kostin, T. Boettger, T. Kubin, T. Braun, E. Bober, Sirt7 increases stress resistance of cardiomyocytes and prevents apoptosis and inflammatory cardiomyopathy in mice. Circ. Res. 102, 703-710 (2008).

28. L. Li, L. Shi, S. Yang, R. Yan, D. Zhang, J. Yang, L. He, W. Li, X. Yi, L. Sun, J. Liang, Z. Cheng, L. Shi, Y. Shang, W. Yu, SIRT7 is a histone desuccinylase that functionally links to chromatin compaction and genome stability. Nat. Commun. 7, 12235 (2016).

29. Y. Ramazani, N. Knops, M. A. Elmonem, T. Q. Nguyen, F. O. Arcolino, L. van den Heuvel, E. Levtchenko, D. Kuypers, R. Goldschmeding, Connective tissue growth factor (CTGF) from basics to clinics. Matrix Biol. 68-69, 44-66 (2018).

30. M. Buschbeck, I. Uribesalgo, I. Wibowo, P. Rué, D. Martin, A. Gutierrez, L. Morey, R. Guigó, H. López-Schier, L. Di Croce, The histone variant macroH2A is an epigenetic regulator of key developmental genes. Nat. Struct. Mol. Biol. 16, 1074-1079 (2009).

31. C. Ito, Y. Saito, T. Nozawa, S. Fujii, T. Sawa, H. Inoue, T. Matsunaga, S. Khan, S. Akashi, R. Hashimoto, C. Aikawa, E. Takahashi, H. Sagara, M. Komatsu, K. Tanaka, T. Akaike, I. Nakagawa, H. Arimoto, Endogenous nitrated nucleotide is a key mediator of autophagy and innate defense against bacteria. Mol. Cell 52, 794-804 (2013).

32. V. Sica, L. Galluzzi, J. M. Bravo-San Pedro, V. Izzo, M. C. Maiuri, G. Kroemer, Organelle-specific initiation of autophagy. Mol. Cell 59, 522-539 (2015).

33. M. Bagherniya, A. E. Butler, G. E. Barreto, A. Sahebkar, The effect of fasting or calorie restriction on autophagy induction: A review of the literature. Ageing Res. Rev. 47, 183-197 (2018).

34. S. Imai, C. M. Armstrong, M. Kaeberlein, L. Guarente, Transcriptional silencing and longevity protein Sir2 is an NAD-dependent histone deacetylase. Nature 403, 795-800 (2000).

35. D. Moazed, Enzymatic activities of Sir2 and chromatin silencing. Curr. Opin. Cell Biol. 13, 232-238 (2001).
36. M. Altmeyer, S. Messner, P. O. Hassa, M. Fey, M. O. Hottiger, Molecular mechanism of poly(ADP-ribosyl)ation by PARP1 and identification of lysine residues as ADP-ribose acceptor sites. Nucleic Acids Res. 37, 3723-3738 (2009).

37. U. M. Muthurajan, M. R. D. Hepler, A. R. Hieb, N. J. Clark, M. Kramer, T. Yao, K. Luger, Automodification switches PARP-1 function from chromatin architectural protein to histone chaperone. Proc. Natl. Acad. Sci. U.S.A. 111, 12752-12757 (2014).

38. K. Ouararhni, R. Hadj-Slimane, S. Ait-Si-Ali, P. Robin, F. Mietton, A. Harel-Bellan, S. Dimitrov, A. Hamiche, The histone variant $\mathrm{mH} 2 \mathrm{~A} 1.1$ interferes with transcription by down-regulating PARP-1 enzymatic activity. Genes Dev. 20, 3324-3336 (2006).

39. S. Chen, J. Seiler, M. Santiago-Reichelt, K. Felbel, I. Grummt, R. Voit, Repression of RNA polymerase I upon stress is caused by inhibition of RNA-dependent deacetylation of PAF53 by SIRT7. Mol. Cell 52, 303-313 (2013).

40. L. Sun, G. Fan, P. Shan, X. Qiu, S. Dong, L. Liao, C. Yu, T. Wang, X. Gu, Q. Li, X. Song, L. Cao, $X$. Li, Y. Cui, S. Zhang, C. Wang, Regulation of energy homeostasis by the ubiquitinindependent REG $\gamma$ proteasome. Nat. Commun. 7, 12497 (2016).

41. W. R. Swindell, Genes and gene expression modules associated with caloric restriction and aging in the laboratory mouse. BMC Genomics 10, 585 (2009).

42. X. Wen, C. Qian, Y. Zhang, R. Wu, L. Lu, C. Zhu, X. Cheng, R. Cui, H. You, F. Mei, J. Gao, F. Li, L. Bu, S. Qu, Key pathway and gene alterations in the gastric mucosa associated with obesity and obesity-related diabetes. J. Cell. Biochem. 120, 6763-6771 (2019).

43. D. Stacey, L. G. Ciobanu, B. T. Baune, A systematic review on the association between inflammatory genes and cognitive decline in non-demented elderly individuals. Eur. Neuropsychopharmacol. 27, 568-588 (2017).

44. H. Yu, W. Ye, J. Wu, X. Meng, R.-Y. Liu, X. Ying, Y. Zhou, H. Wang, C. Pan, W. Huang, Overexpression of sirt7 exhibits oncogenic property and serves as a prognostic factor in colorectal cancer. Clin. Cancer Res. 20, 3434-3445 (2014).

45. J. Yu, B. Qin, F. Wu, S. Qin, S. Nowsheen, S. Shan, J. Zayas, H. Pei, Z. Lou, L. Wang, Regulation of serine-threonine kinase Akt activation by NAD+-dependent deacetylase SIRT7. Cell Rep. 18, 1229-1240 (2017).

46. Z. Ungvari, M. N. Valcarcel-Ares, S. Tarantini, A. Yabluchanskiy, G. A. Fülöp, T. Kiss, A. Csiszar, Connective tissue growth factor (CTGF) in age-related vascular pathologies. GeroScience 39, 491-498 (2017).

47. H. Whitton, L. N. Singh, M. A. Patrick, A. J. Price, F. G. Osorio, C. López-Otín, I. M. Bochkis, Changes at the nuclear lamina alter binding of pioneer factor Foxa2 in aged liver. Aging Cell 17, e12742 (2018).

48. K. Luger, T. J. Rechsteiner, T. J. Richmond, Expression and purification of recombinant histones and nucleosome reconstitution. Methods Mol. Biol. 119, 1-16 (1999).

49. V. Bilan, M. Leutert, P. Nanni, C. Panse, M. O. Hottiger, Combining higher-energy collision dissociation and electron-transfer/higher-energy collision dissociation fragmentation in a product-dependent manner confidently assigns proteomewide ADP-ribose acceptor sites. Anal. Chem. 89, 1523-1530 (2017).

50. I. A. Hendriks, S. C. Larsen, M. L. Nielsen, An advanced strategy for comprehensive profiling of ADP-ribosylation sites using mass spectrometry-based proteomics. Mol. Cell. Proteomics 18, 1010-1026 (2019).

Acknowledgments: We thank A. Ladurner and F. Söllner (LMU, Munich) for support and advice on ADP-ribosylation. We are grateful to the members of the Vaquero laboratory for stimulating discussions. We also thank H. F. Willard, E. Verdin, and J. Gurdon for sharing reagents; J. J. Bech for proteomics bioinformatic analysis; and L. Pardo for access to computing facilities. Funding: This work was supported by the Spanish Ministry of Economy and Competitiveness (MINECO) (SAF2011-25860, SAF2014-55964R, and SAF2017-88975R to A.V.; SAF2016-77830R to M.O.; and CTQ2016-80364-P to E.S.) and cofunded by FEDER funds/European Regional Development Fund (ERDF)-A Way to Build Europe, the Catalan government agency AGAUR (2014-SGR400 and 2017-SGR148 to A.V. and 2017-SGR595 to E.S.), a grant from Rutgers Human Genetics Institute of New Jersey (J.T. and L.S.), the German Center for Cardiovascular Research (DZHK), and the Deutsche Forschungsgemeinschaft (DFG SFB TRR81 A02 and SFB 1213 TP B02 to A.I. and T.B.). We also thank the CERCA Programme/ Generalitat de Catalunya for institutional support. Proteomic analyses were performed in the IDIBELL and CRG Proteomic Units, both of which are part of ProteoRed PRB3 and are supported by grant PT17/0019 from the PE I+D+i 2013-2016, funded by ISCIII and ERDF. Author contributions: N.G.S. and A.V. conceived the study and designed the experiments. N.G.S. performed most of the experiments. A.V. supervised the experiments, coordinated the work, and wrote the paper. J.K.T, B.N.V., J.T., and L.S. generated the SirT7 KO MEFS and performed and analyzed the ChIP-seq and RNA-seq experiments and the aging mouse model. M.E.-A. provided support for the ChIP and qPCR experiments. M.O. performed all the structural analyses. A.I. and T.B. carried out the $C R$ procedure and provided KO MEFs and reagents. J.M.-S., E.S., and C.D.L.T. did the MS/MS analyses. M.B. and S.H.-B. provided reagents and expertise. M.E. participated in the design, generation, and analysis of the RNA-seq experiments. All authors 
participated in the discussions leading to the production of this paper. Competing interests: The authors declare that they have no competing interests. Data and materials availability: All data needed to evaluate the conclusions in the paper are present in the paper and/or the Supplementary Materials. All sequencing data for ChIP-seq and RNA-seq that support the findings of this study have been deposited in the National Center for Biotechnology Information Gene Expression Omnibus (GEO) and are accessible through the GEO Series accession number GSE128894. Proteomics data are available in Pride with the dataset identifier PXD018344 and in table S1. Additional data related to this paper may be requested from the authors.
Submitted 30 August 2019

Accepted 4 June 2020

Published 24 July 2020

$10.1126 /$ sciadv.aaz2590

Citation: N. G. Simonet, J. K. Thackray, B. N. Vazquez, A. Ianni, M. Espinosa-Alcantud J. Morales-Sanfrutos, S. Hurtado-Bagès, E. Sabidó, M. Buschbeck, J. Tischfield, C. De La Torre, M. Esteller, T. Braun, M. Olivella, L. Serrano, A. Vaquero, SirT7 auto-ADP-ribosylation regulates glucose starvation response through mH2A1. Sci. Adv. 6, eaaz2590 (2020). 